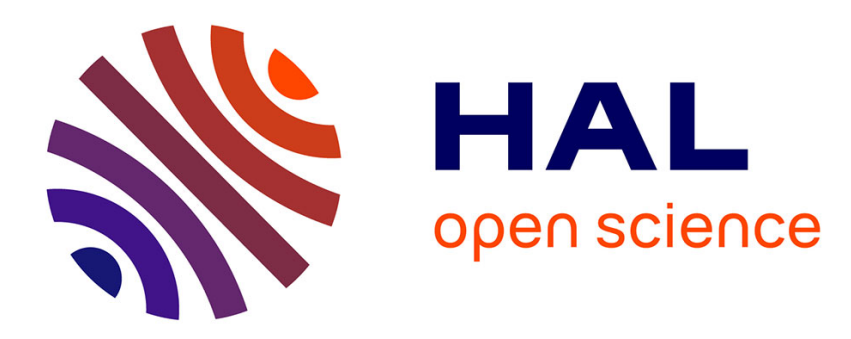

\title{
Asymptotic modeling of thin piezoelectric plates
}

Thibaut Weller, Christian Licht

\section{To cite this version:}

Thibaut Weller, Christian Licht. Asymptotic modeling of thin piezoelectric plates. Annals of Solid and Structural Mechanics, 2010, 2 (2-4), pp.87-98. 10.1007/s12356-010-0013-1 . hal-00742903

\section{HAL Id: hal-00742903 \\ https://hal.science/hal-00742903}

Submitted on 17 Oct 2012

HAL is a multi-disciplinary open access archive for the deposit and dissemination of scientific research documents, whether they are published or not. The documents may come from teaching and research institutions in France or abroad, or from public or private research centers.
L'archive ouverte pluridisciplinaire HAL, est destinée au dépôt et à la diffusion de documents scientifiques de niveau recherche, publiés ou non, émanant des établissements d'enseignement et de recherche français ou étrangers, des laboratoires publics ou privés. 


\title{
ASYMPTOTIC MODELING OF THIN PIEZOELECTRIC PLATES
}

\author{
Thibaut Weller, Christian Licht \\ Laboratoire de Mécanique et Génie Civil, UMR-CNRS 5508, \\ c.c. 048, Université Montpellier II, Place Eugène Bataillon \\ 34095 Montpellier Cedex 5, France \\ \{weller, licht\}@lmgc.univ-montp2.fr
}

\begin{abstract}
We study the asymptotic behavior of a three dimensional flat, heterogeneous and anisotropic piezoelectric body when its thickness - seen as a parameter - goes to zero. Depending on the type of the electrical loading two models are obtained which are related to the plate used as a sensor or as an actuator. These models are explicitly derived in any piezoelectric crystal symmetry class. For some of them, a striking structural switch-off of the piezoelectric effect occurs. The static case is solved through a unifying approach using techniques of singular perturbation while the transient situation is formulated in terms of evolution equations in Hilbert spaces of possible states with finite electromechanical energy, so that the study of these transient problems are easily deduced from the static case through the Trotter theory of convergence of semi-groups of operators acting on variable spaces.
\end{abstract}

\section{Introduction}

The interest of an efficient modeling of the dynamic response of piezoelectric plates lies in the fact that a major technological application of piezoelectric effect is the control of vibrations of structures through very thin patches. But, if many studies have been devoted to their static behavior (see for instance the introduction in [2]), much less attention have been paid to the dynamic response. We are only aware of the substantial but rather complex modeling of [11]. It seems to us that this complexity stems from both the taking into account of the magnetic effects and its mathematical treatment. The latter involves a variational evolution equation in terms of auxiliary variables like the electrical and the magnetic vector potentials and not in terms of the root variables, i.e. the electric and magnetic fields. Moreover, because of the large discrepancy between the celerities of the mechanical and electromechanical waves, magnetic effects can indeed be ignored. That is why we propose a modeling in the appropriate framework of the quasi-electrostatic approximation through the theory of semi-groups of linear operators acting on variable spaces. Since the Trotter result of convergence of semi-groups claims that the study of the convergence of the transient problems reduces to the static case, we have choosed to first revisit the static modeling of thin piezoelectric plates in an unified way. To this aim, we extend the mathematical derivation of the asymptotic behavior of a linearly elastic plate exposed in [4] to the linearly piezoelectric case. The keypoint is to consider that the thickness of the flat piezoelectric body is a parameter. We study the behavior of the solution of the physical problem when this parameter goes to zero. Our modeling is derived from the limit behavior, so that the thinner the plate the sharper the modeling. It is also efficient from the computational point of view because it involves two-dimensional problems. Indeed, depending on the boundary conditions, two models are obtained which correspond to the cases when the plate acts as a sensor or as an actuator. Because the piezoelectric effect is significantly increased in some composite materials, our motivation is to treat realistic situations (general heterogeneous and anisotropic piezoelectric materials) in order that our mathematical analysis be applicable, even if some of its aspects have already been studied in some papers for unrealistic cases (see Section 3.1).

\section{The static case}

As usual we make no difference between the physical space and $\mathbb{R}^{3}$ and, for all $\xi=\left(\xi_{1}, \xi_{2}, \xi_{3}\right)$ in $\mathbb{R}^{3}$, we define

$$
\widehat{\xi}:=\left(\xi_{1}, \xi_{2}\right) .
$$

In all this paper, greek indices for coordinates take their values in 1,2 whereas latin indices run from 1 to 3 . Let

$$
\mathcal{H}:=S^{3} \times \mathbb{R}^{3},
$$

where $S^{3}$ denotes the set of all $3 \times 3$ real and symmetric matrices. For the sake of simplicity, the classical symbol ' . ' will stand for the scalar product in $\mathcal{H}, S^{3}$ and $\mathbb{R}^{3}$. The set of all linear mappings from a space $V$ into a space $W$ is denoted 
T. Weller \& C. Licht

$\mathcal{L}(V, W)$. If $V=W$, we simply write $\mathcal{L}(V)$. In the sequel, for every domain $G$ in $\mathbb{R}^{N}$, the subset of the Sobolev space $H^{1}(G)$ whose elements vanish on $\Gamma$, included in the boundary $\partial G$ of $G$, will be denoted by:

$$
H_{\Gamma}^{1}(G):=\left\{v \in H^{1}(G): v_{\mid \Gamma}=0, \Gamma \subset \partial G\right\}
$$

\subsection{Setting the problem}

The reference configuration of a linearly piezoelectric thin plate is the closure in $\mathbb{R}^{3}$ of the set $\Omega^{\varepsilon}:=\omega \times(-\varepsilon, \varepsilon)$, where $\varepsilon$ is a small positive number and $\omega$ a bounded domain of $\mathbb{R}^{2}$ with a Lipschitz boundary $\partial \omega$. The lateral part of the plate $\partial \omega \times(-\varepsilon, \varepsilon)$ is denoted $\Gamma_{\text {lat }}^{\varepsilon}$, while the set constituted by its lower and upper faces is $\Gamma_{ \pm}^{\varepsilon}:=\omega \times\{ \pm \varepsilon\}$. Let $\left(\Gamma_{m D}^{\varepsilon}, \Gamma_{m N}^{\varepsilon}\right)$, $\left(\Gamma_{e D}^{\varepsilon}, \Gamma_{e N}^{\varepsilon}\right)$ two suitable partitions of $\partial \Omega^{\varepsilon}$ with both $\Gamma_{m D}^{\varepsilon}$ and $\Gamma_{e D}^{\varepsilon}$ of strictly positive Lebesgue measure. The plate is, on one hand, clamped along $\Gamma_{m D}^{\varepsilon}$ and at an electric potential $\varphi_{0}^{\varepsilon}$ on $\Gamma_{e D}^{\varepsilon}$ and, on the other hand, subjected to body forces $f^{\varepsilon}$ and electrical loadings $F^{\varepsilon}$ in $\Omega^{\varepsilon}$. Actually $F^{\varepsilon}$ vanishes, the material being an insulator, anyway the following analysis stands with $F^{\varepsilon}$ different from 0 . Moreover, the plate is subjected to surface forces $g^{\varepsilon}$ and electrical loadings $d^{\varepsilon}$ on $\Gamma_{m N}^{\varepsilon}$ and $\Gamma_{e N}^{\varepsilon}$ respectively. We note $n^{\varepsilon}$ the outward unit normal to $\partial \Omega^{\varepsilon}$ and assume that $\Gamma_{m D}^{\varepsilon}=\gamma_{0} \times(-\varepsilon, \varepsilon)$, with $\gamma_{0} \subset \partial \omega$. Then the equations determining the electromechanical state $s^{\varepsilon}:=\left(u^{\varepsilon}, \varphi^{\varepsilon}\right)$ at equilibrium are:

$$
\left\{\begin{array}{l}
\operatorname{div} \sigma^{\varepsilon}+f^{\varepsilon}=0 \text { in } \Omega^{\varepsilon}, \sigma^{\varepsilon} n^{\varepsilon}=g^{\varepsilon} \text { on } \Gamma_{m N}^{\varepsilon}, u^{\varepsilon}=0 \text { on } \Gamma_{m D}^{\varepsilon}, \\
\operatorname{div} D^{\varepsilon}+F^{\varepsilon}=0 \text { in } \Omega^{\varepsilon}, D^{\varepsilon} \cdot n^{\varepsilon}=d^{\varepsilon} \text { on } \Gamma_{e N}^{\varepsilon}, \varphi^{\varepsilon}=\varphi_{0}^{\varepsilon} \text { on } \Gamma_{e D}^{\varepsilon} \\
\left(\sigma^{\varepsilon}, D^{\varepsilon}\right)=M^{\varepsilon}(x)(e(u), \nabla \varphi) \text { in } \Omega^{\varepsilon}
\end{array}\right.
$$

where $u^{\varepsilon}, \varphi^{\varepsilon}, \sigma^{\varepsilon}, e\left(u^{\varepsilon}\right)$ and $D^{\varepsilon}$ respectively denote the displacement and electrical potential fields, the stress tensor, the linearized strain tensor and the electrical displacement. In the linearly piezoelectric framework which is studied here, we recall that the operator $M^{\varepsilon}$ is an element of $\mathcal{L}(\mathcal{H})$ such that:

$$
\left\{\begin{array}{l}
\sigma^{\varepsilon}=M_{m m}^{\varepsilon} e\left(u^{\varepsilon}\right)-M_{m e}^{\varepsilon} \nabla \varphi^{\varepsilon}, \\
D^{\varepsilon}=M_{m e}^{\varepsilon^{T}} e\left(u^{\varepsilon}\right)+M_{e e}^{\varepsilon} \nabla \varphi^{\varepsilon}
\end{array}\right.
$$

where $\left(M_{m m}^{\varepsilon}, M_{m e}^{\varepsilon}, M_{e e}^{\varepsilon}\right) \in \mathcal{L}\left(S^{3}\right) \times \mathcal{L}\left(\mathbb{R}^{3}, S^{3}\right) \times \mathcal{L}\left(\mathbb{R}^{3}\right)$ are respectively the elastic, piezoelectric and dielectric tensors while $M_{m e}^{\varepsilon^{T}}=: M_{e m}^{\varepsilon}$ denotes the transpose of $M_{m e}^{\varepsilon}$. We recall that $M_{m m}^{\varepsilon}$ and $M_{e e}^{\varepsilon}$ are symmetric and positive.

To give a variational formulation of (4), we first make the following regularity hypothesis on the exterior loading:

$$
\left(\mathbf{H}_{1}\right):\left\{\begin{array}{l}
\left(f^{\varepsilon}, g^{\varepsilon}, F^{\varepsilon}, d^{\varepsilon}\right) \in L^{2}\left(\Omega^{\varepsilon}\right)^{3} \times L^{2}\left(\Gamma_{m N}^{\varepsilon}\right)^{3} \times L^{2}\left(\Omega^{\varepsilon}\right) \times L^{2}\left(\Gamma_{e N}^{\varepsilon}\right), \\
\varphi_{0}^{\varepsilon} \text { has an } H^{1}\left(\Omega^{\varepsilon}\right)^{3} \text { extension into } \Omega^{\varepsilon} \text { still denoted by } \varphi_{0}^{\varepsilon}
\end{array}\right.
$$

and on the space of electromechanical states

$$
\mathbf{V}^{\varepsilon}:=\left\{r=(v, \psi) \in H_{\Gamma_{m D}^{\varepsilon}}^{1}\left(\Omega^{\varepsilon}\right)^{3} \times H_{\Gamma_{e D}^{\varepsilon}}^{1}\left(\Omega^{\varepsilon}\right)\right\}
$$

we define a bilinear form $m^{\varepsilon}$ :

$$
\left.m^{\varepsilon}(r, q)=m^{\varepsilon}((v, \psi),(w, \phi)):=\int_{\Omega^{\varepsilon}} M^{\varepsilon}(e(v), \nabla \psi)\right) \cdot(e(w), \nabla \phi) d x^{\varepsilon},
$$

and a linear form $L^{\varepsilon}$ :

$$
L^{\varepsilon}(r)=L^{\varepsilon}((v, \psi)):=\int_{\Omega^{\varepsilon}}\left(f^{\varepsilon} \cdot v+F^{\varepsilon} \psi\right) d x^{\varepsilon}+\int_{\Gamma_{m N}^{\varepsilon}} g^{\varepsilon} \cdot v d s^{\varepsilon}+\int_{\Gamma_{e N}^{\varepsilon}} d^{\varepsilon} \psi d s^{\varepsilon} .
$$

The physical problem, set on the real plate, then takes the form

$$
\mathcal{P}\left(\Omega^{\varepsilon}\right) \quad: \quad \text { Find } s^{\varepsilon}=\left(u^{\varepsilon}, \varphi^{\varepsilon}\right) \in\left(0, \varphi_{0}^{\varepsilon}\right)+\mathbf{V}^{\varepsilon} \text { such that } m^{\varepsilon}\left(s^{\varepsilon}, r\right)=L^{\varepsilon}(r), \quad \forall r \in \mathbf{V}^{\varepsilon} .
$$

Thus, with the additional and realistic assumptions of boundedness of $M_{m m}^{\varepsilon}, M_{m e}^{\varepsilon}, M_{e e}^{\varepsilon}$ and of uniform ellipticity of $M_{m m}^{\varepsilon}$ and $M_{e e}^{\varepsilon}$ :

$$
\left(\mathbf{H}_{2}\right) \quad: \quad M^{\varepsilon} \in L^{\infty}\left(\Omega^{\varepsilon}, \mathcal{L}(\mathcal{H})\right), \exists \kappa^{\varepsilon}>0: M^{\varepsilon}\left(x^{\varepsilon}\right) h \cdot h \geq \kappa^{\varepsilon}|h|_{\mathcal{H}}^{2}, \forall h \in \mathcal{H} \text {, a.e. } x^{\varepsilon} \in \Omega^{\varepsilon},
$$

the theorem of Stampacchia (cf. [3]) implies the 
Theorem 1. Under assumptions $\left(\boldsymbol{H}_{1}\right)-\left(\boldsymbol{H}_{2}\right)$, the problem $\mathcal{P}\left(\Omega^{\varepsilon}\right)$ has a unique solution.

To derive a simplified and accurate model, the very question is to study the behavior of $s^{\varepsilon}$ when $\varepsilon$, considered as a parameter, tends to zero. We will show that, depending on the type of boundary conditions, two limit behaviors, indexed by $p$ with value 1 or 2 , can be obtained (see [17]).

\subsection{The scaling operation}

Classically (see [4]), we come down to a fixed open set $\Omega:=\omega \times(-1,1)$ through the mapping $\pi^{\varepsilon}$ :

$$
x=\left(\widehat{x}, x_{3}\right) \in \bar{\Omega} \mapsto \pi^{\varepsilon} x=\left(\widehat{x}, \varepsilon x_{3}\right) \in \overline{\Omega^{\varepsilon}} .
$$

Also, we drop the index $\varepsilon$ for the image by $\left(\pi^{\varepsilon}\right)^{-1}$ of the previous geometric sets. To get physically meaningful results, we have to make various kinds of assumptions. They deal with

1. the electromechanical coefficients:

$\left(\mathbf{H}_{3}\right) \quad: \quad M^{\varepsilon}\left(\pi^{\varepsilon} x\right)=M(x)$ with $M \in L^{\infty}(\Omega, \mathcal{L}(\mathcal{H})), \exists \kappa>0: M(x) h \cdot h \geq \kappa|h|_{\mathcal{H}}^{2}, \forall h \in \mathcal{H}$, a.e. $x \in \Omega$,

2. the electromechanical loading:

$$
\left(\mathbf{H}_{4}\right):\left\{\begin{array}{l}
\text { there exists }(f, F, g, d) \in L^{2}(\Omega)^{3} \times L^{2}(\Omega) \times L^{2}\left(\Gamma_{m N}\right)^{3} \times L^{2}\left(\Gamma_{e N}\right) \\
f_{\alpha}^{\varepsilon}\left(\pi^{\varepsilon} x\right)=\varepsilon f_{\alpha}(x), f_{3}^{\varepsilon}\left(\pi^{\varepsilon} x\right)=\varepsilon^{2} f_{3}(x), F^{\varepsilon}\left(\pi^{\varepsilon} x\right)=\varepsilon^{2-p} F(x), \forall x \in \Omega, \\
g_{\alpha}^{\varepsilon}\left(\pi^{\varepsilon} x\right)=\varepsilon^{2} g_{\alpha}(x), g_{3}^{\varepsilon}\left(\pi^{\varepsilon} x\right)=\varepsilon^{3} g_{3}(x), \forall x \in \Gamma_{m N} \cap \Gamma_{ \pm}, \\
g_{\alpha}^{\varepsilon}\left(\pi^{\varepsilon} x\right)=\varepsilon g_{\alpha}(x), g_{3}^{\varepsilon}\left(\pi^{\varepsilon} x\right)=\varepsilon^{2} g_{3}(x), \forall x \in \Gamma_{m N} \cap \Gamma_{l a t} \\
d^{\varepsilon}\left(\pi^{\varepsilon} x\right)=\varepsilon^{3-p} d(x), \forall x \in \Gamma_{e N} \cap \Gamma_{ \pm}, d^{\varepsilon}\left(\pi^{\varepsilon} x\right)=\varepsilon^{2-p} d(x), \forall x \in \Gamma_{e N} \cap \Gamma_{l a t}, \\
\varphi_{0}^{\varepsilon}\left(\pi^{\varepsilon} x\right)=\varepsilon^{p} \varphi_{0}(x), \forall x \in \Gamma_{e D} .
\end{array}\right.
$$

3. the boundedness of the "work of the exterior loading":

$$
\left(\mathbf{H}_{5}\right):\left\{\begin{aligned}
& p=1: \text { the extension of } \varphi_{0} \text { into } \Omega \text { does not depend on } x_{3} . \\
& p=2: \text { the closure } \bar{\delta} \text { of the projection of } \Gamma_{e D} \text { on } \omega \text { coincides with } \bar{\omega} \\
& \text { and either } d=0 \text { on } \Gamma_{e N} \cap \Gamma_{\text {lat }} \text { or } \Gamma_{e N} \cap \Gamma_{l a t}=\emptyset
\end{aligned}\right.
$$

Also, we associate a scaled electromechanical state $s_{p}(\varepsilon):=\left(u_{p}(\varepsilon), \varphi_{p}(\varepsilon)\right)=: \Pi_{p}^{\varepsilon} s^{\varepsilon}$ defined on $\Omega$ with the true physical electromechanical state $s^{\varepsilon}=\left(u^{\varepsilon}, \varphi^{\varepsilon}\right)$ defined on $\Omega^{\varepsilon}$ :

$$
\widehat{u^{\varepsilon}}\left(x^{\varepsilon}\right)=\varepsilon\left(\widehat{u_{p}(\varepsilon)}\right)(x), u_{3}^{\varepsilon}\left(x^{\varepsilon}\right)=\left(u_{p}(\varepsilon)\right)_{3}(x), \varphi^{\varepsilon}\left(x^{\varepsilon}\right)=\varepsilon^{p} \varphi_{p}(\varepsilon)(x), \forall x^{\varepsilon}=\pi^{\varepsilon} x \in \overline{\Omega^{\varepsilon}} .
$$

Assumptions $\left(\mathbf{H}_{3}\right),\left(\mathbf{H}_{4}\right)$ together with the scaling operation (10) are classical. Actually, they are justified by the convergence results they lead to. If we just consider the displacement, these hypotheses are the ones made in [4] and supply a mathematical justification of the Kirchhoff-Love theory of thin linearly elastic plates.

Remark 1. In the following, if $\mathbf{E}$ stands for any function space defined on $\Omega$, the same function space - but defined on $\Omega^{\varepsilon}$ will be denoted by $\mathbf{E}^{\varepsilon}$ and vice-versa.

\subsection{Variational formulation of the scaled problem}

Let $\mathbf{V}$ be the space of scaled electromechanical states:

$$
\mathbf{V}:=\left\{r=(v, \psi) \in H_{\Gamma_{m D}}^{1}(\Omega)^{3} \times H_{\Gamma_{e D}}^{1}(\Omega)\right\}
$$

Of course, $r \in \mathbf{V}^{\varepsilon} \Longleftrightarrow \Pi_{p}^{\varepsilon} r \in \mathbf{V}$. Now, for all $r=(v, \psi) \in \mathbf{V}$, we define the scaled strain tensor $e(\varepsilon, v)$ and the scaled electrical potential gradient $\nabla_{(p)}(\varepsilon, \psi)$ by:

$$
e_{\alpha \beta}(\varepsilon, v):=e_{\alpha \beta}(v), e_{\alpha 3}(\varepsilon, v):=\varepsilon^{-1} e_{\alpha 3}(v), e_{33}(\varepsilon, v):=\varepsilon^{-2} e_{33}(v), \nabla_{(p)}(\varepsilon, \psi)_{\alpha}:=\varepsilon^{p-1} \partial_{\alpha} \psi, \nabla_{(p)}(\varepsilon, \psi)_{3}:=\varepsilon^{p-2} \partial_{3} \psi
$$


To simplify the notations, we set

$$
k(r):=(e(v), \nabla \psi), \quad k_{p}(\varepsilon, r):=\left(e(\varepsilon, v), \nabla_{(p)}(\varepsilon, \psi)\right),
$$

and, as in (7) and (8) we introduce a bilinear form $m_{p}(\varepsilon)$ and a linear form $L$ on $\mathbf{V}$ :

$$
m_{p}(\varepsilon)(r, q):=\int_{\Omega} M(x) k_{p}(\varepsilon, r) \cdot k_{p}(\varepsilon, q) d x, \quad L(r):=\int_{\Omega}(f \cdot v+F \psi) d x+\int_{\Gamma_{m N}} g \cdot v d s+\int_{\Gamma_{e N}} d \psi d s
$$

so that under asumptions $\left(\mathbf{H}_{1}\right)-\left(\mathbf{H}_{4}\right)$, the scaled electromechanical state $s_{p}(\varepsilon)=\left(u_{p}(\varepsilon), \varphi_{p}(\varepsilon)\right)$ is the unique solution of the mathematical problem:

$$
\mathcal{P}(\varepsilon, \Omega)_{p} \quad: \quad \text { Find } s_{p}(\varepsilon) \in\left(0, \varphi_{0}\right)+\mathbf{V} \text { such that } m_{p}(\varepsilon)\left(s_{p}(\varepsilon), r\right)=L(r), \forall r \in \mathbf{V} .
$$

\subsection{Asymptotic behavior of the scaled electromechanical state}

\section{The process}

To generalize the method described in [4], we will show that some components of $k_{p}\left(\varepsilon, s_{p}(\varepsilon)\right)$ and of $M k_{p}\left(\varepsilon, s_{p}(\varepsilon)\right)$ have vanishing limits when $\varepsilon$ goes to 0 . In the purely mechanical case, we recall that the classical result is that the limit displacement $v$ is of Kirchhoff-Love type, i.e. such that $e_{i 3}(v)=0$. In fact it is possible to generalize this idea to multiphysical couplings by observing that a fundamental role is played by the algebraic structure of the space $\mathcal{H}$ defined in $(2)$ : with the scaling operation, various powers of $\varepsilon$ appear in $k_{p}(\varepsilon,$.$) (see (13)$ ); their signs suggest a suitable orthogonal decomposition of $\mathcal{H}$ in the following subspaces:

$$
\begin{aligned}
& \mathcal{H}_{1}^{-}:=\left\{h=(e, g): e_{\alpha \beta}=0, g_{\alpha}=0\right\}, \mathcal{H}_{2}^{-}:=\left\{h=(e, g): e_{\alpha \beta}=0, g_{i}=0\right\} \text {, } \\
& \mathcal{H}_{1}^{0}:=\left\{h=(e, g): e_{i 3}=0, g_{3}=0\right\} \quad, \mathcal{H}_{2}^{0}:=\left\{h=(e, g): e_{i 3}=0, g_{\alpha}=0\right\} \text {, } \\
& \mathcal{H}_{1}^{+}:=\left\{h=(e, g): e_{i j}=0, g_{i}=0\right\} \quad, \mathcal{H}_{2}^{+}:=\left\{h=(e, g): e_{i j}=0, g_{3}=0\right\} \text {. }
\end{aligned}
$$

This process is similar to the one of [14]. For a given $p \in\{1,2\}, M$ can then be decomposed in nine elements $M_{p}^{\star \diamond} \in \mathcal{L}\left(\mathcal{H}_{p}^{\diamond}, \mathcal{H}_{p}^{\star}\right)$ with $\star, \diamond \in\{-, 0,+\}$. Hypothesis $\left(\mathbf{H}_{3}\right)$ on the electromechanical coefficients implies that $M_{p}^{00}$ et $M_{p}^{--}$are positive operators on $\mathcal{H}_{p}^{0}$ and $\mathcal{H}_{p}^{-}$. Therefore, the Schur complement

$$
\widetilde{M}_{p}:=M_{p}^{00}-M_{p}^{0-}\left(M_{p}^{--}\right)^{-1} M_{p}^{-0}
$$

is an element of $\mathcal{L}\left(\mathcal{H}_{p}^{0}\right)$. It is important to note that neither $M_{p}^{00}$ nor $\widetilde{M}_{p}$ are necessarily symmetric, but nevertheless

$$
\kappa\left|h_{p}^{0}\right|_{\mathcal{H}}^{2} \leqslant \widetilde{M}_{p}(x) h_{p}^{0} \cdot h_{p}^{0}, \quad \forall h_{p}^{0} \in \mathcal{H}_{p}^{0}, \quad \text { a.e. } x \in \Omega .
$$

This is implied by the coercivity of $M\left(\right.$ see $\left.\left(\mathbf{H}_{3}\right)\right)$ and by the fundamental relation:

$$
(M h)_{p}^{-}=h_{p}^{+}=0 \Rightarrow\left\{\begin{array}{l}
\widetilde{M}_{p} h_{p}^{0}=(M h)_{p}^{0}, \\
\widetilde{M}_{p} h_{p}^{0} \cdot h_{p}^{0}=M h \cdot h .
\end{array}\right.
$$

The key point of the asymptotic study is to show that if $\bar{k}_{p}$ is the limit (in a suitable topology) of $k_{p}\left(\varepsilon, s_{p}(\varepsilon)\right.$ ), then $\left(M \bar{k}_{p}\right)_{p}^{-}=\left(\bar{k}_{p}\right)_{p}^{+}=0$ ! This will enable us to exhibit $\widetilde{M}_{p}$ as the operator governing the limit constitutive equations.

\section{Functional framework}

We will show that the limit displacements live in the space $\mathbf{V}_{K L}$ of Kirchhoff-Love displacements

$$
\mathbf{V}_{K L}:=\left\{v \in H_{\Gamma_{m D}}^{1}(\Omega)^{3}: e_{i 3}(v)=0\right\},
$$

while the limit electrical potential belongs to

$$
\Phi_{1}:=\left\{\psi \in H_{\Gamma_{e D}}^{1}(\Omega): \partial_{3} \psi=0\right\} \text { or } \Phi_{2}:=\left\{\psi \in H_{\partial_{3}}^{1}(\Omega): \psi_{\mid \Gamma_{e D} \cap \Gamma_{ \pm}} \equiv 0\right\}
$$


We recall that for all $v \in \mathbf{V}_{K L}$, there exists a unique couple $\left(v^{M}, v^{F}\right) \in H_{\gamma_{0}}^{1}(\omega)^{2} \times H_{\gamma_{0}}^{2}(\omega)$ such that:

$$
\widehat{v}(x)=v^{M}(\widehat{x})-x_{3} \widehat{\nabla} v^{F}(\widehat{x}), \quad v_{3}(x)=v^{F}(\widehat{x}) .
$$

and we introduce

$$
\mathbf{V}_{K L}^{M}:=\left\{v \in \mathbf{V}_{K L}: v^{F}=0\right\}, \quad \mathbf{V}_{K L}^{F}:=\left\{v \in \mathbf{V}_{K L}: v^{M}=0\right\}
$$

The space

$$
H_{\partial_{3}}^{1}(\Omega):=\left\{\psi \in L^{2}(\Omega): \partial_{3} \psi \in L^{2}(\Omega)\right\}
$$

equipped with the scalar product:

$$
(\varphi, \psi) \mapsto \int_{\Omega} \varphi \psi d x+\int_{\Omega} \partial_{3} \varphi \partial_{3} \psi d x
$$

is an Hilbert space. The trace mapping being linear and continuous from $H_{\partial_{3}}^{1}(\Omega)$ to $L^{2}\left(\Gamma_{ \pm}\right)$, the definition $(20)$ of $\Phi_{2}$ is meaningful. Thus, with the assumption $\left(\mathbf{H}_{5}\right), \Phi_{2}$ can be equipped with the scalar product:

$$
(\varphi, \psi) \mapsto \int_{\Omega} \partial_{3} \varphi \partial_{3} \psi d x
$$

equivalent to the one defined by (24). Finally, let

$$
\mathbf{S}_{p}:=\mathbf{V}_{K L} \times \Phi_{p}, \quad \mathbf{X}_{p}:=\left\{\begin{array}{l}
H_{\Gamma_{m D}}^{1}(\Omega)^{3} \times H^{1}(\Omega), \text { if } p=1 \\
H_{\Gamma_{m D}}^{1}(\Omega)^{3} \times H_{\partial 3}^{1}(\Omega), \text { if } p=2
\end{array}\right.
$$

The Korn and Poincaré inequalities allow us to define on $\mathbf{S}_{p}$ and $\mathbf{X}_{p}$ the hilbertian norms

$$
\begin{array}{ll}
|(v, \psi)|_{\mathbf{S}_{1}}^{2}:=|e(v)|_{L^{2}(\Omega)}^{2}+|\nabla \psi|_{L^{2}(\Omega)}^{2}, & ,|(v, \psi)|_{\mathbf{S}_{2}}^{2}:=|e(v)|_{L^{2}(\Omega)}^{2}+\left|\partial_{3} \psi\right|_{L^{2}(\Omega)}^{2} \\
|(v, \psi)|_{\mathbf{X}_{1}}^{2}:=|e(v)|_{L^{2}(\Omega)}^{2}+|\psi|_{L^{2}(\Omega)}^{2}+|\nabla \psi|_{L^{2}(\Omega)}^{2},|(v, \psi)|_{\mathbf{X}_{2}}^{2}:=|e(v)|_{L^{2}(\Omega)}^{2}+|\psi|_{L^{2}(\Omega)}^{2}+\left|\partial_{3} \psi\right|_{L^{2}(\Omega)}^{2}
\end{array}
$$

The set $\left(0, \varphi_{0}\right)+\mathbf{S}_{p}$ will appear to be the limit set of electromechanical states.

Remark 2. Because $k(r)$ is not rigorously defined when $r=(v, \psi)$ belongs to $\mathbf{X}_{2}$ (see definitions (13), (15), (23) and (26)), we are led to slighty abuse of the notations by letting

$$
k(r)_{2}^{0}=\left(\left(e_{\alpha \beta}(v), 0\right),\left(0, \partial_{3} \psi\right)\right) .
$$

\section{The two limit scaled problems}

We have the following convergence result:

Proposition 1. Under assumptions $\left(\boldsymbol{H}_{3}\right)-\left(\boldsymbol{H}_{5}\right)$, and when $\varepsilon \rightarrow 0$, the family $\left(s_{p}(\varepsilon)\right)_{\varepsilon>0}$ of the unique solutions of $\mathcal{P}(\varepsilon, \Omega)_{p}$ strongly converges in $\boldsymbol{X}_{p}$ to the unique solution $\bar{s}_{p}$ of:

$$
\overline{\mathcal{P}}(\Omega)_{p}: \text { Find } s \in\left(0, \varphi_{0}\right)+\boldsymbol{S}_{p} \text { such that } \widetilde{m}_{p}(s, r):=\int_{\Omega} \widetilde{M}_{p} k(s)_{p}^{0} \cdot k(r)_{p}^{0} d x=L(r), \forall r \in \boldsymbol{S}_{p} .
$$

Furthermore, $\lim _{\varepsilon \rightarrow 0} m_{p}(\varepsilon)\left(s_{p}(\varepsilon), s_{p}(\varepsilon)\right)=\widetilde{m}_{p}\left(\bar{s}_{p}, \bar{s}_{p}\right)$. 
Proof. It is divided in five steps.

First step : the family $\left(s_{p}(\varepsilon)\right)_{\varepsilon}$ is bounded in $\mathbf{X}_{p}$.

We may assume $\varepsilon \leq 1$. For all $r \in \mathbf{V}, \widetilde{s}_{p}(\varepsilon):=s_{p}(\varepsilon)-\left(0, \varphi_{0}\right)$ satisfies:

$$
m_{p}(\varepsilon)\left(\widetilde{s}_{p}(\varepsilon), r\right)=\widetilde{L}(r):=\int_{\Omega}(f \cdot v+F \psi) d x+\int_{\Gamma_{m N}} g \cdot v d s+\int_{\Gamma_{e N}} d \psi d s-m_{p}(\varepsilon)\left(\left(0, \varphi_{0}\right), r\right) .
$$

Thus, assumption $\left(\mathbf{H}_{3}\right)$ on the electromechanical coefficients implies:

$$
\left.\kappa \mid \widetilde{s}_{p}(\varepsilon)\right)\left.\right|_{\mathbf{X}_{p}} ^{2} \leq \kappa\left|k\left(\varepsilon, \widetilde{s}_{p}(\varepsilon)\right)\right|_{L^{2}(\Omega, \mathcal{H})}^{2} \leq m_{p}(\varepsilon)\left(\widetilde{s}_{p}(\varepsilon), \widetilde{s}_{p}(\varepsilon)\right)=\widetilde{L}\left(\widetilde{s}_{p}(\varepsilon)\right) .
$$

To establish the boundedness of $\widetilde{s}_{p}(\varepsilon)$ in $\mathbf{X}_{p}$, it suffices to show that there exists a constant $c$ which does not depend on $\varepsilon$ such that

$$
\widetilde{L}\left(\widetilde{s}_{p}(\varepsilon)\right) \leq c\left|k\left(\varepsilon, \widetilde{s}_{p}(\varepsilon)\right)\right|_{L^{2}(\Omega, \mathcal{H})}
$$

Bounding the four terms of $\widetilde{L}\left(\widetilde{s}_{p}(\varepsilon)\right)$ - linked to the work of the exterior loading - is obvious except the third in the case $p=2$ and the fourth if $p=1$. Indeed, $\left(\mathbf{H}_{5}\right)$ implies $\frac{1}{\varepsilon} \partial_{3} \varphi_{0}=0$, therefore $\left|k_{1}\left(\varepsilon,\left(0, \varphi_{0}\right)\right)\right|_{L^{2}(\Omega)}$ is uniformly bounded with respect to $\varepsilon$; assumption $\left(\mathbf{H}_{5}\right)$ deletes the problem of bounding $\int_{\Gamma_{e N}} d\left(\varphi(\varepsilon)-\varphi_{0}\right) d s$. Hence, $\varphi_{0}$ being fixed, the family $\left(s_{p}(\varepsilon)\right)_{0<\varepsilon \leq 1}$ is bounded in the Hilbert space $\mathbf{X}_{p}$ and so there exists a subsequence, not relabelled, such that:

$$
\begin{aligned}
\left(s_{p}(\varepsilon), k\left(\varepsilon, s_{p}(\varepsilon)\right)\right. & \rightarrow\left(\bar{s}_{p}, \bar{k}_{p}\right) \text { in } \mathbf{X}_{p} \times L^{2}(\Omega, \mathcal{H}), \\
k\left(s_{p}(\varepsilon)\right)_{p}^{-} & \rightarrow 0 \text { in } L^{2}(\Omega, \mathcal{H}), \\
k\left(\bar{s}_{p}\right)_{p}^{0} & =\left(\bar{k}_{p}\right)_{p}^{0}
\end{aligned}
$$

where the $\rightarrow$ and $\rightarrow$ symbols respectively denote weak and strong convergences.

Second step : to identify the operator providing the limit constitutive law, we establish that $\left(M \bar{k}_{p}\right)_{p}^{-}=\left(\bar{k}_{p}\right)_{p}^{+}=0$.

To get $\left(M \bar{k}_{p}\right)_{p}^{-}=0$, we generalize the method introduced in [4] in the case of linearly isotropic elastic plates. In the equation associated with $\mathcal{P}(\varepsilon, \Omega)_{p}$, we choose:

i) $r$ such that $v_{3}=\psi=0$ and multiply by $\varepsilon$,

ii) $r$ such that $v_{\alpha}=0$ and $\psi=0$ and multiply by $\varepsilon^{2}$,

iii) $r$ such $v=0$ and multiply by $\varepsilon$ if $p=1$ only,

and, by going to the limit, we conclude with the lemma of [4]:

Lemma 1. If $w \in L^{2}(\Omega)$ satisfies $\int_{\Omega} w \partial_{3} v d x=0$, for all $v$ in $C^{\infty}(\bar{\Omega})$ such that $v=0$ on $\gamma \times[-1,1]$, then $w=0$.

From its very definition $\left(\bar{k}_{1}\right)_{1}^{+}$vanishes, while $\left(\bar{k}_{2}\right)_{2}^{+}=0$ stems from a classical argument (see [15] for the details) in going to the limit in the identity

$$
\int_{\Omega} \varepsilon \partial_{\alpha} \varphi(\varepsilon) g d x=-\varepsilon \int_{\Omega} \varphi(\varepsilon) \partial_{\alpha} g d x, \forall g \in C_{0}^{\infty}(\Omega)
$$

Therefore, using (18), we have

$$
M \bar{k}_{p} \cdot \bar{k}_{p}=\widetilde{M}\left(\bar{k}_{p}\right)_{p}^{0} \cdot\left(\bar{k}_{p}\right)_{p}^{0}, \quad\left(M \bar{k}_{p}\right)_{p}^{0}=\widetilde{M}_{p}\left(\bar{k}_{p}\right)_{p}^{0}
$$

Third step : the limit problem.

For all $r \in \mathbf{S}_{p} \cap C^{\infty}\left(\bar{\Omega}, \mathbb{R}^{4}\right), k_{p}(\varepsilon, r)$ strongly converges to $k(r)_{p}^{0}$ in $L^{2}(\Omega, \mathcal{H})$. Therefore, by passing to the limit in $\mathcal{P}(\varepsilon, \Omega)_{p}$ with (32), (36) and (34), we get

$$
L(r)=\int_{\Omega} M \bar{k}_{p} \cdot k(r)_{p}^{0} d x=\int_{\Omega}\left(M \bar{k}_{p}\right)_{p}^{0} \cdot k(r)_{p}^{0} d x=\int_{\Omega} \widetilde{M}_{p}\left(\bar{k}_{p}\right)_{p}^{0} \cdot k(r)_{p}^{0} d x=\int_{\Omega} \widetilde{M}_{p} k\left(\bar{s}_{p}\right)_{p}^{0} \cdot k(r)_{p}^{0} d x
$$


The density of $\mathbf{S}_{p} \cap C^{\infty}\left(\bar{\Omega}, \mathbb{R}^{4}\right)$ in $\mathbf{S}_{p}$ implies that $\bar{s}_{p}$ solves $\mathcal{P}(0, \Omega)_{p}$. The bilinear form $\widetilde{m}_{p}$ being $\mathbf{S}_{p}$-elliptic (see (17)), the problem $\mathcal{P}(0, \Omega)_{p}$ has a unique solution. Thus, by a classical compacity argument, the whole sequence $s_{p}(\varepsilon)$ weakly converges to $\bar{s}_{p}$.

Fourth step : strong convergence.

$\overline{\mathrm{By}(32)-(34)}$, the strong convergence of $s_{p}(\varepsilon)$ to $\bar{s}_{p}$ in $\mathbf{X}_{p}$ is equivalent to the one of $k\left(s_{p}(\varepsilon)\right)_{p}^{0}$ to $k\left(s_{p}\right)_{p}^{0}$ in $L^{2}(\Omega, \mathcal{H})$. But we have

$$
\begin{aligned}
& \kappa\left|k\left(s_{p}(\varepsilon)\right)_{p}^{0}-k\left(\bar{s}_{p}\right)_{p}^{0}\right|_{L^{2}(\Omega, \mathcal{H})}^{2} \leq \kappa\left|k\left(\varepsilon, s_{p}(\varepsilon)\right)-\bar{k}_{p}\right|_{L^{2}(\Omega, \mathcal{H})}^{2} \leq \int_{\Omega} M\left(k\left(\varepsilon, s_{p}(\varepsilon)\right)-\bar{k}_{p}\right) \cdot\left(k\left(\varepsilon, s_{p}(\varepsilon)\right)-\bar{k}_{p}\right) d x \\
& =\int_{\Omega} M\left(k\left(\varepsilon, s_{p}(\varepsilon)\right)\right) \cdot\left(k\left(\varepsilon, s_{p}(\varepsilon)\right)\right) d x-\int_{\Omega} M \bar{k}_{p} \cdot\left(k\left(\varepsilon, s_{p}(\varepsilon)\right) d x-\int_{\Omega} M\left(k\left(\varepsilon, s_{p}(\varepsilon)\right)-\bar{k}_{p}\right) \cdot \bar{k}_{p} d x\right. \\
& \quad=L\left(\widetilde{s}_{p}(\varepsilon)\right)+\int_{\Omega} M\left(k\left(\varepsilon, s_{p}(\varepsilon)\right)\right) \cdot\left(k\left(\varepsilon,\left(0, \varphi_{0}\right)\right)\right) d x-\int_{\Omega} M \bar{k}_{p} \cdot\left(k\left(\varepsilon, s_{p}(\varepsilon)\right) d x-\int_{\Omega} M\left(k\left(\varepsilon, s_{p}(\varepsilon)\right)-\bar{k}_{p}\right) \cdot \bar{k}_{p} d x\right.
\end{aligned}
$$

and from (32) and (36), we deduce that, as $\varepsilon \rightarrow 0$, the right member converges to

$l=L\left(\bar{s}_{p}-\left(0, \varphi_{0}\right)\right)+\int_{\Omega} M \bar{k}_{p} \cdot k\left(0, \varphi_{0}\right) d x-\int_{\Omega} M \bar{k}_{p} \cdot \bar{k}_{p} d x=L\left(\bar{s}_{p}-\left(0, \varphi_{0}\right)\right)+\int_{\Omega} \widetilde{M}_{p} \bar{k}_{p}^{0} \cdot k\left(0, \varphi_{0}\right)_{p}^{0} d x-\int_{\Omega} \widetilde{M}_{p} \bar{k}_{p}^{0} \cdot \bar{k}_{p}^{0} d x=0$.

Fifth step : $\lim _{\varepsilon \rightarrow 0} m_{p}(\varepsilon)\left(s_{p}(\varepsilon), s_{p}(\varepsilon)\right)=\widetilde{m}_{p}\left(\bar{s}_{p}, \bar{s}_{p}\right)$.

It suffices to observe that $(32),\left(\mathbf{H}_{5}\right)$ and (36) imply

$$
\lim _{\varepsilon \rightarrow 0} m_{p}(\varepsilon)\left(s_{p}(\varepsilon),\left(0, \varphi_{0}\right)\right)=\int_{\Omega} M \bar{k}_{p} \cdot k\left(0, \varphi_{0}\right)_{p}^{0} d x=\widetilde{m}_{p}\left(\bar{s}_{p},\left(0, \varphi_{0}\right)\right)
$$

\subsection{Variants to Proposition 1}

It is interesting to consider the cases when the elements $f, F, g, d$ and $\varphi_{0}$ are depending on $\varepsilon$ or when the linear form $L$ defined in (14) is a more abstract mathematical object than the electromechanical loading. In this direction, a careful examination of the preceding proof leads to the

Corollary 1. Let $\left(\Lambda_{\varepsilon}\right)_{\varepsilon>0}$ a family of continuous linear forms on $\boldsymbol{X}_{p}$ which converges weakly to $\Lambda$ and $\left(\varphi_{0_{\varepsilon}}\right)_{\varepsilon>0} \subset H^{1}(\Omega)$ such that

$$
\left(\boldsymbol{H}_{5}\right)^{\prime}:\left\{\begin{array}{l}
\exists \varphi_{0} \in H^{1}(\Omega) \text { if } p=1, H_{\partial 3}^{1}(\Omega) \text { if } p=2, \exists k_{0} \in L^{2}(\Omega, \mathcal{H}) \\
\left(\varphi_{0_{\varepsilon}}, k\left(\varepsilon, \varphi_{0_{\varepsilon}}\right)\right) \rightarrow\left(\varphi_{0}, k_{0}\right) \text { in } L^{2}(\Omega) \times L^{2}(\Omega, \mathcal{H}) \text { with }\left(k_{0}\right)_{p}^{0}=\left(k\left(0, \varphi_{0}\right)\right)_{p}^{0},
\end{array}\right.
$$

then, when $\varepsilon \rightarrow 0$, the family $\left(s_{p}^{\prime}(\varepsilon)\right)_{\varepsilon>0}$ of the unique solutions of

Find $s \in\left(0, \varphi_{0_{\varepsilon}}\right)+\boldsymbol{V}$ such that $m_{p}(\varepsilon)(s, r)=\Lambda_{\varepsilon}(r), \forall r \in \boldsymbol{V}$.

weakly converges in $\boldsymbol{X}_{p}$ to the unique solution $\bar{s}_{p}^{\prime}$ of

$$
\text { Find } s \in\left(0, \varphi_{0}\right)+\boldsymbol{S}_{p} \text { such that } \widetilde{m}_{p}(s, r)=\Lambda(r), \forall r \in \boldsymbol{S}_{p}
$$

In addition, if the previous convergences of the data are strong then the familly $\left(s_{p}^{\prime}(\varepsilon)\right)_{\varepsilon>0}$ converges strongly in $\boldsymbol{X}_{p}$ and

$$
\lim _{\varepsilon \rightarrow 0} m_{p}(\varepsilon)\left(s_{p}^{\prime}(\varepsilon), s_{p}^{\prime}(\varepsilon)\right)=\widetilde{m}_{p}\left(\bar{s}_{p}^{\prime}, \bar{s}_{p}^{\prime}\right) .
$$

For instance, when $\Lambda_{\varepsilon}$ is the scaled work:

$$
\Lambda_{\varepsilon}(r)=L_{\varepsilon}(r)=\int_{\Omega}\left(f_{\varepsilon} \cdot v+F_{\varepsilon} \psi\right) d x+\int_{\Gamma_{m N}} g_{\varepsilon} \cdot v d s+\int_{\Gamma_{e N}} d_{\varepsilon} \psi d s,
$$


T. Weller \& C. Licht

with $d_{\varepsilon}=0$ on $\Gamma_{e N} \cap \Gamma_{l a t}$ or $\Gamma_{e N} \cap \Gamma_{l a t}=\emptyset$ if $p=2$, the weak convergence of $\Lambda_{\varepsilon}$ is implied by

$$
\left(f_{\varepsilon}, F_{\varepsilon}, g_{\varepsilon}, d_{\varepsilon}\right) \rightarrow(f, F, g, d) \text { in } L^{2}(\Omega)^{3} \times L^{2}(\Omega) \times L^{2}\left(\Gamma_{m N}\right)^{3} \times L^{2}\left(\Gamma_{e N}\right) .
$$

Actually, when $p=1$, this assumption yields the strong convergence of $\Lambda_{\varepsilon}$ which is obtained if $p=2$ with the additional assumption of strong convergence of $\left(F_{\varepsilon}, d_{\varepsilon}\right)$ in $L^{2}(\Omega) \times L^{2}\left(\Gamma_{e N}\right)$. It is worthwile to note that $\left(\mathbf{H}_{5}^{\prime}\right)$ implies the weak $($ resp. strong) convergence of $\varphi_{0_{\varepsilon}}$ in $H^{1}(\Omega)$ with $\partial_{3} \varphi_{0}=0$ if $p=1$ or in $H_{\partial 3}^{1}(\Omega)$ with $\left(k_{0}\right)_{p}^{+}=0$ if $p=2$.

It is also interesting to consider a perturbation of the bilinear form $m_{p}(\varepsilon)$. In fact, the following corollary will play a crucial role in the study of the dynamic case. Let us introduce the assumption

$$
\left(\mathbf{H}_{p}^{\text {decoupl }}\right): \int_{-1}^{+1} x_{3} \widetilde{M}_{1} d x_{3}=0, \quad \widetilde{M}_{2}\left(\widehat{x}, x_{3}\right)=\widetilde{M}_{2}(\widehat{x}) \text { with } \Gamma^{ \pm} \subset \Gamma_{e D}
$$

and the bilinear forms

$$
\begin{aligned}
(v, w) & \in L^{2}(\Omega)^{2} \mapsto k_{l}(\varepsilon)(v, w):=\varepsilon^{2(l-1)} \int_{\Omega}\left(\widehat{v} \cdot \widehat{w}+\varepsilon^{-2} v_{3} w_{3}\right) d x, l=1,2, \\
(v, w) \in \mathbf{V}_{K L}^{M^{2}} & \mapsto \quad \widetilde{k}_{1}(v, w):=\int_{\Omega} \widehat{v} \cdot \widehat{w} d x, \\
(v, w) \in \mathbf{V}_{K L}^{F^{2}} & \mapsto \quad \widetilde{k}_{2}(v, w):=\int_{\Omega} v_{3} w_{3} d x,
\end{aligned}
$$

and let

$$
\mathbf{V}_{1}:=\mathbf{V}_{K L}^{M}, \quad \mathbf{W}_{1}:=\mathbf{V}_{K L}^{F}, \quad \mathbf{V}_{2}:=\mathbf{V}_{K L}^{F}, \quad \mathbf{W}_{2}:=\mathbf{V}_{K L}^{M}
$$

We have the

Corollary 2. Let $\lambda \in \mathbb{R}^{+}$and $\Lambda$ a continuous linear form on $\boldsymbol{X}_{p}$ such that $\Lambda\left(\boldsymbol{W}_{l}\right)=0$. Then, under assumptions ( $\left.\boldsymbol{H}_{3}\right)$ and $\left(\boldsymbol{H}_{p}^{\text {decoupl }}\right)$, the familly $\left(s_{p, l}^{\prime \prime}(\varepsilon)\right)_{\varepsilon>0}$ of the unique solutions of

$$
\text { Find } s=(u, \varphi) \in \boldsymbol{V} \text { such that } k_{l}(\varepsilon)(u, w)+\lambda m_{p}(\varepsilon)(s, r)=\Lambda(r), \forall r=(w, \psi) \in \boldsymbol{V},
$$

converges strongly in $\boldsymbol{X}_{p}$ to the unique solution $\bar{s}_{p, l}^{\prime \prime}$ of

$$
\text { Find } s=(u, \phi) \in \boldsymbol{V}_{l} \times \Phi_{p} \text { such that } \widetilde{k}_{l}(u, w)+\lambda \widetilde{m}_{p}(s, r)=\Lambda(r), \forall r=(w, \psi) \in \boldsymbol{V}_{l},
$$

and, of course,

$$
\lim _{\varepsilon \rightarrow 0} k_{l}(\varepsilon)\left(u_{p, l}^{\prime \prime}(\varepsilon), u_{p, l}^{\prime \prime}(\varepsilon)\right)+\lambda m_{p}(\varepsilon)\left(s_{p, l}^{\prime \prime}(\varepsilon), s_{p, l}^{\prime \prime}(\varepsilon)\right)=\widetilde{k}_{l}(\varepsilon)\left(\bar{u}_{p, l}^{\prime \prime}, \bar{u}_{p, l}^{\prime \prime}\right)+\lambda \widetilde{m}_{p}\left(\bar{s}_{p, l}^{\prime \prime}, \bar{s}_{p, l}^{\prime \prime}\right)
$$

Actually, the additional property $\bar{s}_{p, l}^{\prime \prime} \in \mathbf{V}_{l}$ is deduced from assumption $\left(\mathbf{H}_{p}^{\text {decoupl }}\right)$ which permits (see section 2.7) to exploit $\Lambda\left(\mathbf{W}_{l}\right)=0$.

\subsection{Back to the problem $\mathcal{P}\left(\Omega^{\varepsilon}\right)$ : a proposal of simplified and acurate modeling}

We now come back to the reference configuration $\overline{\Omega^{\varepsilon}}$ of the real plate of thickness $2 \varepsilon$ through the operators $\pi^{\varepsilon}$ and $\left(\Pi_{p}^{\varepsilon}\right)^{-1}$ (see (9) and (10)). With the solution $\bar{s}_{p}$ of $\overline{\mathcal{P}}(\Omega)_{p}$ is associated a physical electromechanical state $\bar{s}_{p}^{\varepsilon}$ defined on $\Omega^{\varepsilon}$ by:

$$
\bar{s}_{p}^{\varepsilon}\left(\pi^{\varepsilon} x\right):=\left(\Pi_{p}^{\varepsilon}\right)^{-1} \bar{s}_{p}(x), \forall x \in \Omega
$$

This electromechanical state is the solution of a problem posed over $\Omega^{\varepsilon}$ which is the transportation by $\pi^{\varepsilon}$ of the (limit scaled) problem $\overline{\mathcal{P}}(\Omega)_{p}$. This transported problem, set on $\Omega^{\varepsilon}$, is our proposal to model thin linearly piezoelectric plates of thickness $2 \varepsilon$. The function $\bar{s}_{p}^{\varepsilon}$ represents an approximation of the electromechanical state $s^{\varepsilon}$ inside the plate. It remains to show that this approximation is acurate. In this direction, we let

$$
\widetilde{M}_{p}^{\varepsilon}\left(\pi^{\varepsilon} x\right):=\widetilde{M}_{p}(x), \forall x \in \Omega, \quad(s, r) \in \mathbf{V}^{\varepsilon^{2}} \mapsto \widetilde{m}_{p}^{\varepsilon}(s, r):=\int_{\Omega^{\varepsilon}} \widetilde{M}_{p}^{\varepsilon}(x) k(s)_{p}^{0} \cdot k(r)_{p}^{0} d x
$$

Proposition 1 implies the 
Theorem 2. Under assumptions $\left(\boldsymbol{H}_{3}\right)-\left(\boldsymbol{H}_{5}\right)$, the couple $\bar{s}_{p}^{\varepsilon}=:\left(\bar{u}_{p}^{\varepsilon}, \bar{\varphi}_{p}^{\varepsilon}\right)$ constituted by the limit ("descaled") displacement and electrical potential defined over the physical plate $\Omega^{\varepsilon}$ is the unique solution of the problem:

$$
\overline{\mathcal{P}}\left(\Omega^{\varepsilon}\right)_{p} \quad: \quad \text { Find } s \in\left(0, \varphi_{0}^{\varepsilon}\right)+\boldsymbol{S}_{p}^{\varepsilon} \text { such that } \widetilde{m}_{p}^{\varepsilon}(s, r)=L(r), \forall r \in \boldsymbol{S}_{p}^{\varepsilon}
$$

Furthermore, the electromechanical state $\bar{s}_{p}^{\varepsilon}$ is asymptotically equivalent to the unique solution $s^{\varepsilon}$ of the genuine physical problem $\mathcal{P}\left(\Omega^{\varepsilon}\right)$ in the sense that:

$$
\begin{gathered}
\lim _{\varepsilon \rightarrow 0} \varepsilon^{-1} \int_{\Omega^{\varepsilon}} \varepsilon^{-2}\left|\left(\bar{u}_{p}^{\varepsilon}\right)_{\alpha}-u_{\alpha}^{\varepsilon}\right|^{2}+\left|\left(\bar{u}_{p}^{\varepsilon}\right)_{3}-u_{3}^{\varepsilon}\right|^{2} d x^{\varepsilon}=0, \quad \lim _{\varepsilon \rightarrow 0} \varepsilon^{-3} \int_{\Omega^{\varepsilon}}\left|e_{\alpha \beta}^{\varepsilon}\left(\left(\bar{u}_{p}^{\varepsilon}\right)_{\alpha}\right)-e_{\alpha \beta}^{\varepsilon}\left(u^{\varepsilon}\right)\right|^{2} d x^{\varepsilon}=0 \\
\lim _{\varepsilon \rightarrow 0} \varepsilon^{-3} \int_{\Omega^{\varepsilon}}\left|\bar{\varphi}_{1}^{\varepsilon}-\varphi^{\varepsilon}\right|^{2}+\left|\partial_{\alpha}^{\varepsilon} \bar{\varphi}_{1}^{\varepsilon}-\partial_{\alpha}^{\varepsilon} \varphi^{\varepsilon}\right|^{2} d x^{\varepsilon}=0, \quad \lim _{\varepsilon \rightarrow 0} \varepsilon^{-5} \int_{\Omega^{\varepsilon}}\left|\bar{\varphi}_{2}^{\varepsilon}-\varphi^{\varepsilon}\right|^{2}+\varepsilon^{2}\left|\partial_{3}^{\varepsilon} \bar{\varphi}_{2}^{\varepsilon}-\partial_{3}^{\varepsilon} \varphi^{\varepsilon}\right|^{2} d x^{\varepsilon}=0 \\
\varepsilon^{-3} \int_{\Omega^{\varepsilon}}\left|e_{i 3}\left(u^{\varepsilon}\right)\right|^{2} d x^{\varepsilon}, \quad \varepsilon^{-3} \int_{\Omega^{\varepsilon}}\left|\partial_{\alpha}^{\varepsilon} \varphi^{\varepsilon}\right|^{2} d x^{\varepsilon} \quad \text { and } \quad \varepsilon^{-3} \int_{\Omega^{\varepsilon}}\left|\partial_{3}^{\varepsilon} \varphi^{\varepsilon}\right|^{2} d x^{\varepsilon} \quad \text { are bounded. }
\end{gathered}
$$

We emphasize on the following points (see [17]):

1. the first model, with $\varphi_{0}^{\varepsilon}=0$, deals with the physical situation when the plate is used as a sensor,

2. the second model corresponds to an actuator.

\subsection{Bidimensional limit equations. Decoupling}

To simplify the notations (and to be realistic), we make the additional assumption

$$
\left(\mathbf{H}_{6}\right) \quad: \quad F^{\varepsilon}=0 \text { and there exists } \gamma_{e N} \subset \partial \omega \text { such that } \Gamma_{e N}=\gamma_{e N} \times(-1,+1) .
$$

Let $\gamma_{m N}=\gamma \backslash \gamma_{0}$, we consider the functions $g_{i}^{\varepsilon \pm}, d^{\varepsilon \pm}, p_{i}^{\varepsilon}, q_{\alpha}^{\varepsilon}, r_{i}^{\varepsilon}, s_{\alpha}^{\varepsilon}, p^{\prime \varepsilon}, r^{\varepsilon} \in L^{2}(\omega)$ defined by

$$
g_{i}^{\varepsilon \pm}(\widehat{x}):=\left\{\begin{array}{l}
g_{i}^{\varepsilon}(\widehat{x}, \pm \varepsilon) \text { if } x \in \Gamma_{m N}^{\varepsilon} \cap \Gamma_{ \pm}^{\varepsilon} \\
0 \text { in the other cases }
\end{array} \quad, \quad d^{\varepsilon \pm}(\widehat{x}):=\left\{\begin{array}{l}
d^{\varepsilon}(\widehat{x}, \pm \varepsilon) \text { if } x \in \Gamma_{e N}^{\varepsilon} \cap \Gamma_{ \pm}^{\varepsilon} \\
0 \text { in the other cases }
\end{array}\right.\right.
$$

and

$$
\begin{array}{llll}
p_{i}^{\varepsilon}:=\int_{-\varepsilon}^{+\varepsilon} f_{i}^{\varepsilon} d x+g_{i}^{\varepsilon+}+g_{i}^{\varepsilon-}, & q_{\alpha}^{\varepsilon}:=\int_{-\varepsilon}^{+\varepsilon} x f_{\alpha}^{\varepsilon} d x+g_{\alpha}^{\varepsilon+}-g_{\alpha}^{\varepsilon-}, r_{i}^{\varepsilon}:=\int_{-\varepsilon}^{+\varepsilon} g_{i}^{\varepsilon} d x, \\
s_{\alpha}^{\varepsilon}:=\int_{-\varepsilon}^{+\varepsilon} x g_{\alpha}^{\varepsilon} d x & , p^{\prime \varepsilon}:=d^{\varepsilon+}+d^{\varepsilon-} & , r^{\prime \varepsilon}:=\int_{-\varepsilon}^{+\varepsilon} d^{\varepsilon} d x .
\end{array}
$$

\section{Case $p=1$}

We define

$$
\begin{gathered}
L_{K L}^{M^{\varepsilon}}\left(v^{M}\right):=\int_{\omega} p_{\alpha}^{\varepsilon} v_{\alpha}^{M} d \widehat{x}+\int_{\gamma_{m N}}\left[r_{\alpha}^{\varepsilon} v_{\alpha}^{M}-s_{\alpha}^{\varepsilon} v_{\alpha}^{M}\right] d \widehat{x}, \quad L_{K L}^{F^{\varepsilon}}\left(v^{F}\right):=\int_{\omega} p_{3}^{\varepsilon} v^{F} d \widehat{x}-\int_{\omega} q_{\alpha}^{\varepsilon} \partial_{\alpha} v^{F} d \widehat{x}+\int_{\gamma_{m N}} r_{3}^{\varepsilon} v^{F} d \widehat{x}, \\
L_{e}^{\varepsilon}(\psi):=\int_{\omega} p^{\varepsilon \varepsilon} \psi d \widehat{x}-\int_{\gamma_{e N}} r^{\prime \varepsilon} \psi d \widehat{x},
\end{gathered}
$$

for all $v=\left(v^{M}, v^{F}\right) \in \mathbf{V}_{K L}^{\varepsilon}$ and all $\psi \in \Phi_{1}^{\varepsilon}$. The limit space $\mathbf{S}_{1}^{\varepsilon}$ is the direct sum of the two subspaces

$$
\mathbf{S}_{1}^{M^{\varepsilon}}:=\mathbf{V}_{K L}^{M^{\varepsilon}} \times \Phi_{1}^{\varepsilon}, \quad \mathbf{S}_{1}^{F^{\varepsilon}}:=\mathbf{V}_{K L}^{F^{\varepsilon}} \times\{0\},
$$

and for all $r=(v, \psi) \in \mathbf{S}_{1}^{\varepsilon}$, we have

$$
\begin{aligned}
k(r)_{1}^{0}(x)= & {\left[\left(\begin{array}{ccc}
e_{11}\left(v^{M}\right)-x_{3} \partial_{11}^{2} v^{F} & e_{12}\left(v^{M}\right)-x_{3} \partial_{12}^{2} v^{F} & 0 \\
e_{12}\left(v^{M}\right)-x_{3} \partial_{12}^{2} v^{F} & e_{22}\left(v^{M}\right)-x_{3} \partial_{22}^{2} v^{F} & 0 \\
0 & 0 & 0
\end{array}\right),\left(\begin{array}{c}
\partial_{1} \psi \\
\partial_{2} \psi \\
0
\end{array}\right)\right] } \\
= & {\left[\left(\begin{array}{ccc}
e_{11}\left(v^{M}\right) & e_{12}\left(v^{M}\right) & 0 \\
e_{12}\left(v^{M}\right) & e_{22}\left(v^{M}\right) & 0 \\
0 & 0 & 0
\end{array}\right),\left(\begin{array}{c}
\partial_{1} \psi \\
\partial_{2} \psi \\
0
\end{array}\right)\right]-x_{3}\left[\left(\begin{array}{ccc}
\partial_{11}^{2} v^{F} & \partial_{12}^{2} v^{F} & 0 \\
\partial_{12}^{2} v^{F} & \partial_{22}^{2} v^{F} & 0 \\
0 & 0 & 0
\end{array}\right),\left(\begin{array}{l}
0 \\
0 \\
0
\end{array}\right)\right]=: k\left(v^{M}, \psi\right)_{1}^{0}(\widehat{x})-x_{3} D^{2}\left(v^{F}\right)_{1}^{0}(\widehat{x}) . }
\end{aligned}
$$


Letting

$$
\widetilde{\mathcal{M}}_{1}^{0}=\widetilde{\mathcal{M}}_{1}^{0}(\widehat{x}):=\int_{-\varepsilon}^{+\varepsilon} \widetilde{M}_{1}^{\varepsilon}\left(x^{\varepsilon}\right) d x_{3}^{\varepsilon}, \quad \widetilde{\mathcal{M}}_{1}^{1}=\widetilde{\mathcal{M}}_{1}^{1}(\widehat{x}):=\int_{-\varepsilon}^{+\varepsilon} x_{3}^{\varepsilon} \widetilde{M}_{1}^{\varepsilon}\left(x^{\varepsilon}\right) d x_{3}^{\varepsilon}, \quad \widetilde{\mathcal{M}}_{1}^{2}=\widetilde{\mathcal{M}}_{1}^{2}(\widehat{x}):=\int_{-\varepsilon}^{+\varepsilon} x_{3}^{\varepsilon^{2}} \widetilde{M}_{1}^{\varepsilon} d x_{3}^{\varepsilon},
$$

the problem $\overline{\mathcal{P}}\left(\Omega^{\varepsilon}\right)_{1}$ takes the following form

$\overline{\mathcal{P}}\left(\Omega^{\varepsilon}\right)_{1}\left\{\begin{array}{c}\text { Find }(u, \varphi) \in\left(0, \varphi_{0}\right)+\mathbf{S}_{1}^{\varepsilon} \text { such that } \\ \int_{\omega}\left[\widetilde{\mathcal{M}}_{1}^{0} k\left(u^{M}, \varphi\right)_{1}^{0} \cdot k\left(v^{M}, \psi\right)_{1}^{0}-\widetilde{\mathcal{M}}_{1}^{1} k\left(u^{M}, \varphi\right)_{1}^{0} \cdot D^{2}\left(v^{F}\right)_{1}^{0}-\widetilde{\mathcal{M}}_{1}^{1} D^{2}\left(u^{F}\right)_{1}^{0} \cdot k\left(v^{M}, \psi\right)_{1}^{0}+\widetilde{\mathcal{M}}_{1}^{2} D^{2}\left(u^{F}\right)_{1}^{0} \cdot D^{2}\left(v^{F}\right)_{1}^{0}\right] d \widehat{x} \\ =L_{K L}^{M^{\varepsilon}}\left(v^{M}\right)+L_{K L}^{F^{\varepsilon}}\left(v^{F}\right)+L_{e}^{\varepsilon}(\psi), \forall(v, \psi) \in \mathbf{S}_{1}^{\varepsilon} .\end{array}\right.$

It is important to note that $\overline{\mathcal{P}}\left(\Omega^{\varepsilon}\right)_{1}$ is a bidimensional problem in the sense that it is posed over $\omega$ and that $\mathbf{S}_{1}^{\varepsilon}$ only involves functions defined on $\omega$. We then remark that hypothesis $\left(\mathbf{H}_{p}^{\text {decoupl}}\right)$ (which is true if the electromechanical coefficients are even functions of $x_{3}$ ) implies a decoupling between membrane displacements and flexural displacements in the sense that they solve two independent variational equations:

$\overline{\mathcal{P}}\left(\Omega^{\varepsilon}\right)_{1}\left\{\begin{array}{l}\text { Find }\left(u^{M}, \varphi\right) \in\left(0, \varphi_{0}\right)+\mathbf{S}_{1}^{M^{\varepsilon}} \text { such that } \int_{\omega} \widetilde{\mathcal{M}}_{1}^{0} k\left(u^{M}, \varphi\right)_{1}^{0} \cdot k\left(v^{M}, \psi\right)_{1}^{0} d \widehat{x}=L_{K L}^{M^{\varepsilon}}\left(v^{M}\right)+L_{e}^{\varepsilon}(\psi), \forall\left(v^{M}, \psi\right) \in \mathbf{S}_{1}^{M^{\varepsilon}} \\ \text { Find } u^{F} \in \mathbf{V}_{K L}^{F^{\varepsilon}} \text { such that } \int_{\omega} \widetilde{\mathcal{M}}_{1}^{2} D^{2}\left(u^{F}\right)_{1}^{0} \cdot D^{2}\left(v^{F}\right)_{1}^{0} d \widehat{x}=L_{K L}^{F^{\varepsilon}}\left(v^{F}\right), \forall v^{F} \in \mathbf{S}_{1}^{F^{\varepsilon}}\end{array}\right.$

where the second problem does not involve the electrical potential. The decisive aspect of assumption $\left(\mathbf{H}_{p}^{\text {decoupl }}\right)$ is that it implies the $\widetilde{m}_{1}^{\varepsilon}$-polarity of $\mathbf{S}_{1}^{M^{\varepsilon}}$ and $\mathbf{S}_{1}^{F^{\varepsilon}}$, i.e.:

$$
\widetilde{m}_{1}^{\varepsilon}\left(s^{M}, r^{F}\right)=\widetilde{m}_{1}\left(r^{F}, s^{M}\right)=0, \quad \forall\left(s^{M}, r^{F}\right) \in \mathbf{S}_{1}^{M^{\varepsilon}} \times \mathbf{S}_{1}^{F^{\varepsilon}}
$$

\section{Case $p=2$}

From its very definition, $\bar{\varphi}_{2}^{\varepsilon}$ satisfies

$$
\partial_{3}\left(\widetilde{M}_{2 e e}^{\varepsilon} \partial_{3} \bar{\varphi}_{2}^{\varepsilon}\right)=-\partial_{3}\left(\widetilde{M}_{2 m e}^{\varepsilon} \widehat{e}\left(\bar{u}_{2}^{\varepsilon M}\right)(\widehat{x})-x_{3} D^{2}\left(\bar{u}_{2}^{\varepsilon F}\right)(\widehat{x})\right),
$$

so that $\bar{\varphi}_{2}^{\varepsilon}(\widehat{x}, \cdot)$ is a second order polynomial as soon as $\widetilde{M}_{2}^{\varepsilon}$ does not depend on $x_{3}$. When $\widetilde{M}_{2}^{\varepsilon}$ depends on $x_{3}$ but $\Gamma_{ \pm}^{\varepsilon} \subset \Gamma_{e D}^{\varepsilon}$, $\bar{\varphi}_{2}^{\varepsilon}$ is a local function of $\widehat{e}\left(\bar{u}_{2}^{\varepsilon M}\right)$ and $D^{2}\left(\bar{u}_{2}^{\varepsilon F}\right)$ :

$$
\bar{\varphi}_{2}^{\varepsilon}=A^{\varepsilon}\left(\varphi_{0}^{\varepsilon^{+}}(\widehat{x})-\varphi_{0}^{\varepsilon^{-}}(\widehat{x})\right)+B^{\varepsilon} \widehat{e}\left(\bar{u}_{2}^{\varepsilon M}\right)+C^{\varepsilon} D^{2}\left(\bar{u}_{2}^{\varepsilon F}\right),
$$

where

$$
\begin{gathered}
A^{\varepsilon}:=a^{\varepsilon^{-1}}(\widehat{x}, \varepsilon) \partial_{3} a^{\varepsilon}, \quad B^{\varepsilon}:=\partial_{3} b^{\varepsilon}-a^{\varepsilon^{-1}} b^{\varepsilon}(\widehat{x}, \varepsilon) \partial_{3} a^{\varepsilon}, \quad C^{\varepsilon}:=\partial_{3} c^{\varepsilon}-a^{\varepsilon^{-1}} c^{\varepsilon}(\widehat{x}, \varepsilon) \partial_{3} a^{\varepsilon}, \\
a^{\varepsilon}\left(\widehat{x}, x_{3}\right):=\int_{-\varepsilon}^{x_{3}}\left(\widetilde{M}_{2_{e e}}^{\varepsilon}\right)^{-1}(\widehat{x}, z) d z, \quad b^{\varepsilon}\left(\widehat{x}, x_{3}\right):=\int_{-\varepsilon}^{x_{3}}\left(\widetilde{M}_{2_{e e}}^{\varepsilon}\right)^{-1} \widetilde{M}_{2_{m e}}^{\varepsilon^{T}}(\widehat{x}, z) d z \\
\varphi_{0}^{\varepsilon^{ \pm}}(\widehat{x}):=\varphi_{0}^{\varepsilon}(\widehat{x}, \pm \varepsilon), \quad c^{\varepsilon}\left(\widehat{x}, x_{3}\right):=\int_{-\varepsilon}^{x_{3}} z\left(\widetilde{M}_{2_{e e}}^{\varepsilon}\right)^{-1} \widetilde{M}_{2_{m e}}^{\varepsilon^{T}}(\widehat{x}, z) d z .
\end{gathered}
$$

Hence, $\bar{u}_{2}^{\varepsilon}$ satisfies

$$
\begin{aligned}
\int_{\Omega^{\varepsilon}}\left[\widetilde{M}_{2 m m}^{\varepsilon}\left(\widehat{e}\left(u^{\varepsilon M}\right)-x_{3} D^{2}\left(u^{\varepsilon F}\right)\right)+\widetilde{M}_{2 m e}^{\varepsilon}\left(B^{\varepsilon} \widehat{e}\left(u^{\varepsilon M}\right)+C^{\varepsilon} D^{2}\left(u^{\varepsilon F}\right)\right] \cdot\left[\widehat{e}\left(v^{M}\right)-x_{3} D^{2} v^{F}\right] d x\right. \\
=-\int_{\Omega^{\varepsilon}}\left(\widetilde{M}_{2_{m e}}^{\varepsilon} A^{\varepsilon}\left(\varphi^{\varepsilon+}-\varphi^{\varepsilon-}\right)\right) d x+L_{K L}^{M^{\varepsilon}}\left(v^{M}\right)+L_{K L}^{F^{\varepsilon}}\left(v^{F}\right), \forall v \in \mathbf{V}_{K L}^{\varepsilon} .
\end{aligned}
$$

Actually, it is a bidimensionnal non symmetric variational problem set on $\omega^{\varepsilon}$. Moreover, with the additional hypothesis $\left(\mathbf{H}_{2}^{\text {decoupl }}\right)$, there is a decoupling between the membrane and the flexural parts of the displacement. If we let

$$
\Phi_{2}^{o^{\varepsilon}}:=\left\{x_{3} \text {-odd parts of } \Phi_{2}^{\varepsilon} \text { elements }\right\}, \quad \Phi_{2}^{e^{\varepsilon}}:=\left\{x_{3} \text {-even parts of } \Phi_{2}^{\varepsilon} \text { elements }\right\},
$$


the two subspaces

$$
\mathbf{S}_{2}^{M^{\varepsilon}}:=\mathbf{V}_{K L}^{M^{\varepsilon}} \times \Phi_{2}^{o^{\varepsilon}}, \quad \mathbf{S}_{2}^{F^{\varepsilon}}:=\mathbf{V}_{K L}^{F^{\varepsilon}} \times \Phi_{2}^{e^{\varepsilon}},
$$

are $\widetilde{m}_{2}$-polar in the sense of (55) where the index 1 is replaced by 2 .

These facts, presented in [17] and [19], already noted under stronger symmetry hypothesis in [10], [15] and [9], have also been observed latter in [5].

\subsection{Some properties of the limit constitutive laws}

It is interesting to give some properties of the operators $\widetilde{M}_{p}^{\varepsilon}$ which supply the constitutive equations of the piezoelectric plate. For a detailed discussion on this point, see [19]. Similarly to (5), we associate with $\widetilde{M}_{p}^{\varepsilon}$ the sub-operators $\widetilde{M}_{p_{m m}}^{\varepsilon}$, $\widetilde{M}_{p_{e m}}^{\varepsilon}, \widetilde{M}_{p_{m e}}^{\varepsilon}, \widetilde{M}_{p_{e e}}^{\varepsilon}$. Due to the fact that the projections from $\mathcal{H}$ to $\mathcal{H}_{0}^{p}$ commutes with the involution $\operatorname{id}_{S^{3}}-\mathrm{id}_{\mathbb{R}^{3}}$, the fundamental coupling property of $M^{\varepsilon}$ remains true for the Schur complement $\widetilde{M}_{p}^{\varepsilon}$ :

$$
\widetilde{M}_{p_{e m}}^{\varepsilon}=-\left(\widetilde{M}_{p_{m e}}^{\varepsilon}\right)^{T}
$$

A handmade proof of this nice property can be found in [19]. Considering the influence of crystalline symmetries (see for example [13]), we deduce that in the case of a polarization normal to the plate:

- $\widetilde{M}_{2 m m}^{\varepsilon}$ involves mechanical terms only,

- $\widetilde{M}_{1_{m m}}^{\varepsilon}=\widetilde{M}_{2_{m m}}^{\varepsilon}$ for the crystalline classes $m, 32,422, \overline{6}, 622$ and $\overline{6} m 2$,

- $\widetilde{M}_{1 m m}^{\varepsilon}$ involves electrical terms except for these previous classes,

- when $p=1$, there is an electromechanical decoupling $\left(\widetilde{M}_{1_{m e}}^{\varepsilon}=0\right)$ for the classes $2,222,2 m m, 4, \overline{4}, 422,4 m m, \overline{4} 2 m, 6$, $622,6 \mathrm{~mm}$ and 23 , while when $p=2$, this decoupling occurs with the classes $m, 32,422, \overline{6}, 622$ and $\overline{6} m 2$, nevertheless the operators $\widetilde{M}_{p_{m m}}^{\varepsilon}$ and $\widetilde{M}_{p_{e e}}^{\varepsilon}$ involve a mixture of elastic, piezoelectric and dielectric coefficients. In these cases, $\widetilde{M}_{p}^{\varepsilon}$ is symmetric which involves a quadratic convex energy. For plates made of these piezoelectric monocrystals, the piezoelectric effect disappears at the structural level!

\section{Application and example: 222 crystalline class}

Let's consider a thin piezoelectric plate constituted by a material whose crystalline symmetry class is 222 . Then (5) takes the form:

$$
\left(\begin{array}{c}
\sigma_{11} \\
\sigma_{22} \\
\sigma_{33} \\
\sqrt{2} \sigma_{23} \\
\sqrt{2} \sigma_{31} \\
\sqrt{2} \sigma_{12} \\
D_{1} \\
D_{2} \\
D_{3}
\end{array}\right)=\left(\begin{array}{cccccc|ccc}
a_{11} & a_{12} & a_{13} & 0 & 0 & 0 & 0 & 0 & 0 \\
a_{12} & a_{22} & a_{23} & 0 & 0 & 0 & 0 & 0 & 0 \\
a_{13} & a_{23} & a_{33} & 0 & 0 & 0 & 0 & 0 & 0 \\
0 & 0 & 0 & a_{44} & 0 & 0 & -b_{41} & 0 & 0 \\
0 & 0 & 0 & 0 & a_{55} & 0 & 0 & -b_{52} & 0 \\
0 & 0 & 0 & 0 & 0 & a_{66} & 0 & 0 & -b_{63} \\
\hline 0 & 0 & 0 & b_{41} & 0 & 0 & c_{11} & 0 & 0 \\
0 & 0 & 0 & 0 & b_{52} & 0 & 0 & c_{22} & 0 \\
0 & 0 & 0 & 0 & 0 & b_{63} & 0 & 0 & c_{33}
\end{array}\right) \cdot\left(\begin{array}{c}
e_{11}(u) \\
e_{22}(u) \\
e_{33}(u) \\
\sqrt{2} e_{23}(u) \\
\sqrt{2} e_{31}(u) \\
\sqrt{2} e_{12}(u) \\
\varphi, 1 \\
\varphi_{, 2} \\
\varphi, 3
\end{array}\right)
$$

Therefore, (16) leads to

$$
\left(\begin{array}{c}
\sigma_{11} \\
\sigma_{22} \\
\sqrt{2} \sigma_{12} \\
D_{1} \\
D_{2}
\end{array}\right)=\left(\begin{array}{ccc|cc}
a_{11}-\frac{a_{13}^{2}}{a_{33}} & a_{12}-\frac{a_{13} a_{23}}{a_{33}} & 0 & 0 & 0 \\
a_{12}-\frac{a_{13} a_{23}}{a_{33}} & a_{22}-\frac{a_{23}^{2}}{a_{33}} & 0 & 0 & 0 \\
0 & 0 & a_{66}+\frac{b_{63}^{2}}{c_{33}} & 0 & 0 \\
\hline 0 & 0 & 0 & c_{11}+\frac{b_{41}^{2}}{a_{44}} & 0 \\
0 & 0 & 0 & 0 & c_{22}+\frac{b_{52}^{2}}{a_{55}}
\end{array}\right) \cdot\left(\begin{array}{c}
e_{11}(u) \\
e_{22}(u) \\
\sqrt{2} e_{12}(u) \\
\varphi, 1 \\
\varphi_{, 2}
\end{array}\right)
$$

in the sensor case $(p=1)$ and to 


$$
\left(\begin{array}{c}
\sigma_{11} \\
\sigma_{22} \\
\sqrt{2} \sigma_{12} \\
D_{3}
\end{array}\right)=\left(\begin{array}{ccc|c}
a_{11}-\frac{a_{13}^{2}}{a_{33}} & a_{12}-\frac{a_{13} a_{23}}{a_{33}} & 0 & 0 \\
a_{12}-\frac{a_{13} a_{23}}{a_{33}} & a_{22}-\frac{a_{23}^{3}}{a_{33}} & 0 & 0 \\
0 & 0 & a_{66} & -b_{63} \\
0 & 0 & b_{63} & c_{33}
\end{array}\right) \cdot\left(\begin{array}{c}
e_{11}(u) \\
e_{22}(u) \\
\sqrt{2} e_{12}(u) \\
\varphi, 3
\end{array}\right)
$$

in the actuator case $(p=2)$.

As outlined in the preceeding section, the relation (64) shows that $\sigma$ and $D$ respectively depend solely on $e(u)$ and $\nabla \varphi$ when the plate acts as a sensor, so that it can be considered as no more piezoelectric. However, when the same plate acts as an actuator, the piezoelectric coupling does not vanish as it can be seen in (65). Moreover, we observe that the difference between both $M_{p_{\mathrm{mm}}}$ lies in the inplane shear coefficient: if $p=1$, this coefficient is equal to $a_{66}+\frac{b_{63}^{2}}{c_{33}}$ while it is equal to $a_{66}$ if $p=2$. Since the order of magnitude of the permittivity $c_{33}$ is most of the time very low compared to the piezoelectric constants (see [13]), the term $\frac{b_{63}^{2}}{c_{33}}$ cannot be neglected. Therefore, from the purely mechanical point of view, it appears a significative difference between the two models.

\subsection{Bibliographical note}

For almost twenty years, several asymptotic theories have been proposed to deal with piezoelectric plates. They differ in the scaling techniques adopted and their results depend on the type of electric boundary conditions on upper and lower faces, so that some controversies appeared. For example, by mean of asymptotic expansions, the first two orders of an asymptotic theory of thin piezoelectric plates are established in [7]. At the first order, a purely mechanical Kirchhoff-Love theory emerges, while the electric potential satisfies a two-dimensional Poisson-Neumann problem, with an effective dielectric constant accounting for electromechanical coupling. Nevertheless, these results do not agree with the thin plate limit obtained in [2]. In particular, the deflection given by [7] depends on elastic constants and not on piezoelectric and dielectric ones. The procedure used in [2] to derive field equations governing the piezoelectric problem is based on the initial functions method in conjunction with a rescaling of the applied loads. These discrepancies have been first conciliated in the work of [10] who shows, in spite of a somewhat uncorrect mathematical framework, that these two models were in fact dealing with two different physical situations, so that both were true. This distinction, fundamental for technological applications, is not yet taken into account in some papers and has to be emphasized... It seems that the first rigorous and unified derivation of these facts in the full anisotropic and heterogeneous cases has been presented in [17] and [19]. Some observations concerning these facts also appear in [11]. We can check that in the framework of the present paper, the constitutive laws obtained by [7] and [2] are respectively given by $\widetilde{M}_{1}$ and $\widetilde{M}_{2}$ for $6 \mathrm{~mm}$ crystal class. A piezoelectric plate model was also derived by [12] as a zero-curvature shell model. The case $p=1$ has been treated by [7] through formal asymptotic expansions for a $6 m m$ homogeneous material. To deal with the difficulty introduced by the Dirichlet condition on the electrical potential, [6] used Lagrange's multipliers while [1] simply used a change of variable to make the electrical boundary conditions homogeneous. We also find a mathematical treatment of the case $p=1$ in [10]. The case $p=2$ has been formally treated by [2] for a $6 m m$ homogeneous material. Mathematical derivations can be found in [10] , [15] and [9] in less heterogeneous and less anisotropic situations than our present study. It seems that the useful properties indicated in section 2.8 are new.

\section{The dynamic case}

\section{$5 \quad$ First formulation of the problem}

\subsection{The quasi-(electro)static approximation}

As pointed out in the introduction, the magnetic effects can be disregarded so that the study of the dynamic response of the piezoelectric plate may be carried out in the framework of the quasi-electrostatic approximation which claims that the electrical field still derives from an electrical potential (see [13]) for further physical details). We recall that for a treatment of the piezoelectric evolution problem with the magnetic effects, one can refer to [11]. Nevertheless, the relations between the obtained limit models have to be studied.

\subsection{Setting the problem}

Here we consider that the density of the plate at a point $x=\left(\widehat{x}, x_{3}\right)$ is $\rho \delta\left(\widehat{x}, x_{3} / \varepsilon\right)$ with $\delta$ a positive element of $L^{\infty}(\Omega)$ bounded from below on $\Omega$. Whereas the static problem was only parameterized by the thickness $\varepsilon$ of the plate, it is now 
appropriate to consider a couple $\eta:=(\varepsilon, \rho)$ of real and positive parameters. Just as we get the asymptotic static behavior by letting $\varepsilon$ going to 0 , here, the couple of parameters $\eta$ will tend to $\bar{\eta}=(0, \bar{\rho})$, where $\bar{\rho} \in[0,+\infty[$. We will see that the relative behavior of $\varepsilon$ and $\rho$ plays a crucial role.

The electromechanical state of the plate is given by a triplet $y^{\eta}(t):=\left(u^{\eta}, \varphi^{\eta}, v^{\eta}\right)(t)$ of displacement $u^{\eta}(t)$, electric potential $\varphi^{\eta}(t)$ and velocity $v^{\eta}(t):=\dot{u}^{\eta}(t)$ fields. Here, the time $t$ takes his values in $[0, T]$ and we denote the time derivative by an upper dot. Then, the equations describing the evolution of the system are:

$$
\mathcal{Q}_{\eta}\left(\Omega^{\varepsilon}\right)\left\{\begin{array}{l}
\operatorname{div} \sigma^{\eta}+f^{\eta}=\rho \delta \ddot{u}^{\eta} \text { in } \Omega^{\varepsilon}, \sigma^{\eta} n^{\varepsilon}=g^{\eta} \text { on } \Gamma_{m N}^{\varepsilon}, u^{\eta}=0 \text { on } \Gamma_{m D}^{\varepsilon}, \\
\operatorname{div} D^{\eta}+F^{\eta}=0 \text { in } \Omega^{\varepsilon}, D^{\eta} \cdot n^{\varepsilon}=d^{\eta} \text { on } \Gamma_{e N}^{\varepsilon}, \varphi^{\eta}=\varphi_{0}^{\eta} \text { on } \Gamma_{e D}^{\varepsilon}, \\
\left(\sigma^{\eta}, D^{\eta}\right)=M^{\varepsilon}\left(x^{\varepsilon}\right)\left(e\left(u^{\eta}\right), \nabla \varphi^{\eta}\right) \text { in } \Omega^{\varepsilon}, \\
\left(u^{\eta}, v^{\eta}\right)(0)=\left(u^{\eta 0}, v^{\eta 0}\right) \text { given. }
\end{array}\right.
$$

Above and in the continuation, the index $\varepsilon$ only affects the geometrical data and the constitutive law. On the other hand, the stress and displacements fields, the electric field, etc. are indexed by $\eta$. The evolution problem being quasi-electrostatic, the initial conditions are only related to the displacement and velocity. Our goal is to show that $\mathcal{Q}_{\eta}\left(\Omega^{\varepsilon}\right)$ has a unique solution and then to study the behavior of this solution when $\eta$ goes to $\bar{\eta}=(0, \bar{\rho})$.

\section{New formulation of the problem}

Assumptions $\left(\mathbf{H}_{3}\right)-\left(\mathbf{H}_{4}\right)$ as well as the change of unknowns (10) make it possible to reformulate $\mathcal{Q}_{\eta}\left(\Omega^{\varepsilon}\right)$ as a problem denoted $\mathcal{Q}(\eta, \Omega)_{p}$, mathematically equivalent but posed over the fixed set $\Omega=\omega \times(-1,1)$. We then introduce the following spaces

$$
\mathbf{Y}_{\eta}:=H_{\Gamma_{m D}}^{1}(\Omega)^{3} \times H_{\Gamma_{e D}}^{1}(\Omega) \times L^{2}(\Omega)^{3}, \quad \mathbf{S}_{\eta}:=H_{\Gamma_{m D}}^{1}(\Omega)^{3} \times H_{\Gamma_{e D}}^{1}(\Omega), \quad \mathbf{U}_{\eta}:=H_{\Gamma_{m D}}^{1}(\Omega)^{3} \times L^{2}(\Omega)^{3} .
$$

The use of the index $\eta$ will be justified further by the introduction of norms depending on $\eta$. The scaled electromechanical state

$$
y(\eta):=(u(\eta), \varphi(\eta), v(\eta):=\dot{u}(\eta)) \in \mathbf{Y}_{\eta},
$$

and the following couples

$$
s(\eta):=(u(\eta), \varphi(\eta)) \in \mathbf{S}_{\eta}, \quad \mathrm{U}(\eta):=(u(\eta), v(\eta)) \in \mathbf{U}_{\eta},
$$

will have an important role in the treatment of the evolution problem.

An equivalent scalar product, associated with the scaled kinetic energy, is defined on $L^{2}(\Omega)^{3}$ by

$$
<v, w>_{\eta}:=\rho \int_{\Omega}\left(v_{\alpha} w_{\alpha}+\varepsilon^{-2} v_{3} w_{3}\right) \delta(x) d x .
$$

From now on, we consider that the scaled electromechanical loading involved in $\left(\mathbf{H}_{5}\right)$ may depend on $\eta$ and is indexed by $\eta$. We make the assumption

$$
\left(\mathbf{H}_{7}\right):\left(f_{\eta}, g_{\eta}, F_{\eta}, d_{\eta}, \varphi_{0_{\eta}}\right) \in C^{2,1}\left([0, T] ; L^{2}(\Omega)^{3} \times L^{2}\left(\Gamma_{m N}\right)^{3} \times L^{2}(\Omega) \times L^{2}\left(\Gamma_{e N}\right) \times H^{1}(\Omega)\right),
$$

and keep in $\left(\mathbf{H}_{5}\right)$ the part relative to $p=2$ only, so that, introducing the linear form

$$
L_{\eta}(r ; t):=\int_{\Omega} f_{\eta}(t) \cdot w d x+\int_{\Gamma_{m N}} g_{\eta}(t) \cdot w d s+\int_{\Omega} F_{\eta}(t) \psi d x+\int_{\Gamma_{e N}} d_{\eta}(t) \psi d s, \quad \forall r=(w, \psi) \in \mathbf{S}_{\eta},
$$

the scaled problem $\mathcal{Q}(\eta, \Omega)_{p}$ reads as:

$$
\mathcal{Q}(\eta, \Omega)_{p}\left\{\begin{array}{l}
\text { Find } y(\eta) \in\left(0, \varphi_{0_{\eta}}, 0\right)+\mathbf{Y}_{\eta} \text { such that }<\dot{v}(\eta), w>_{\eta}+m_{p}(\varepsilon)(s(\eta), r)=L_{\eta}(r ; t), \forall(r=(w, \psi), t) \in \mathbf{S}_{\eta} \times[0, T] \\
\mathbf{U}(\eta)(0)=\left(u^{0}(\eta), v^{0}(\eta)\right)=: \mathbf{U}^{0}(\eta) \text { given in } \mathbf{U}_{\eta} .
\end{array}\right.
$$


Classicaly, we seek the solution $y(\eta)$ on the form

$$
y(\eta)=y^{e}(\eta)+y^{r}(\eta),
$$

with $y^{e}$ and $y^{r}$ the solutions of the following problems $\mathcal{P}^{e}(\eta, \Omega)_{p}$ and $\mathcal{Q}^{r}(\eta, \Omega)_{p}$, respectively. The problem $\mathcal{P}^{e}(\eta, \Omega)_{p}$ is quasi-static and takes into account the exterior loading:

$$
\mathcal{P}^{e}(\eta, \Omega)_{p} \quad: \quad \text { Find } s^{e}(\eta) \in\left(0, \varphi_{0_{\eta}}\right)+\mathbf{S}_{\eta} \text { such that } m_{p}(\varepsilon)\left(s^{e}(\eta), r\right)=L_{\eta}(r ; t), \forall(r, t) \in \mathbf{S}_{\eta} \times[0, T],
$$

The existence, the unicity, but also the asymptotic behavior of $s^{e}(\eta)$ have been studied in the previous section. Of course, we define $y^{e}(\eta)$ by $(67)$, and $\left(\mathbf{H}_{7}\right)$ implies:

$$
s^{e}(\eta) \in C^{2,1}\left([0, T] ; \mathbf{S}_{\eta}\right) .
$$

On the other hand the problem $\mathcal{Q}^{r}(\eta, \Omega)_{p}$ is an evolution problem:

$\mathcal{Q}^{r}(\eta, \Omega)_{p}\left\{\begin{array}{l}\text { Find } s^{r}(\eta) \in \mathbf{S}_{\eta} \text { such that }<\ddot{u}^{r}(\eta), w>_{\eta}+m_{p}(\varepsilon)\left(s^{r}(\eta), r\right)=-<\ddot{u}^{e}(\eta), w>_{\eta}, \forall(r=(w, \psi), t) \in \mathbf{S}_{\eta} \times[0, T], \\ u^{r}(\eta)(0)=u^{0}(\eta)-u^{e}(\eta)(0), \dot{u}^{r}(\eta)(0)=v^{0}(\eta)-\dot{u}^{e}(\eta)(0) .\end{array}\right.$

Taking into account the true nature of $\mathcal{Q}(\eta, \Omega)_{p}$, quasi-static with respect to the electrical potential and dynamic with respect to the displacement, we can eliminate the electrical potential $\varphi^{r}(\eta)$ whose souvenir is kept by the operator

$$
\mathcal{S}_{\eta}: u \in H_{\Gamma_{m D}}^{1}(\Omega)^{3} \mapsto \mathcal{S}_{\eta} u:=\varphi_{\eta} \in H_{\Gamma_{e D}}^{1}(\Omega) \text { such that } m_{p}(\varepsilon)\left(\left(u, \varphi_{\eta}\right),(0, \psi)\right)=0, \forall \psi \in H_{\Gamma_{e D}}^{1}(\Omega) .
$$

Clearly, from $\left(\mathbf{H}_{2}\right)$ and the Lax-Milgram Lemma, $\mathcal{S}_{\eta}$ is a well defined linear continuous operator. Then, introducing the operator $\mathrm{A}_{\eta}$ :

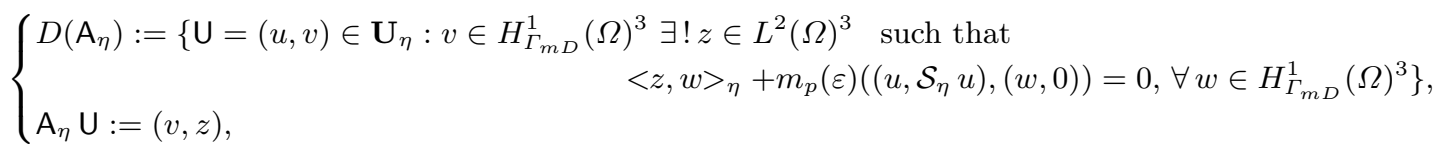

we can associate an evolution equation in $\mathbf{U}_{\eta}$ with the problem $\mathcal{Q}^{r}(\eta, \Omega)_{p}$ :

$$
\dot{\mathrm{U}}^{r}(\eta)-\mathrm{A}_{\eta} \mathrm{U}^{r}(\eta)=\mathrm{F}(\eta):=\left(0,-\ddot{u}^{e}(\eta)\right), \quad \mathrm{U}^{r}(\eta)(0)=\mathrm{U}^{0}(\eta)-\mathrm{U}^{e}(\eta)(0) .
$$

Note that assumption $\left(\mathbf{H}_{7}\right)$ gives a sense to $\mathbf{U}^{r}(\eta)(0)$ and $\mathbf{F}(\eta)$.

With the compatibility assumption between initial loading and mechanical phase

$$
\left(\mathbf{H}_{7}\right): \mathrm{U}^{0}(\eta)-\mathrm{U}^{e}(\eta)(0) \in D\left(\mathrm{~A}_{\eta}\right),
$$

we have the

Proposition 2. Under assumptions $\left(\boldsymbol{H}_{3}\right),\left(\boldsymbol{H}_{4}\right),\left(\boldsymbol{H}_{7}\right)$ and $\left(\boldsymbol{H}_{8}\right)$ the evolution equation (75) has a unique solution of class $C^{1}\left([0, T] ; \boldsymbol{U}_{\eta}\right)$.

Proof. The keypoint is to equip $\mathbf{U}_{\eta}$ by a scalar product $\ll_{.}, \gg_{\eta}$ for which $\mathbf{U}_{\eta}$ is a Hilbert space and $\mathrm{A}_{\eta}$ is skew-adjoint. Let the bilinear continuous form on $\mathbf{U}_{\eta}$ defined by:

$$
\ll \mathrm{U}, \mathrm{U}^{\prime} \gg_{\eta}=\ll(u, v),\left(u^{\prime}, v^{\prime}\right) \gg_{\eta}:=m_{p}(\varepsilon)\left(\left(u, \mathcal{S}_{\eta} u\right),\left(u^{\prime}, 0\right)\right)+<v, v^{\prime}>_{\eta} .
$$

The structure of $M($ see $(5))$ and the very definition of $\mathcal{S}_{\eta}$ imply the symmetry of $\ll_{.}, \gg_{\eta}$ because

$$
\begin{aligned}
m_{p}(\varepsilon)\left(\left(u, \mathcal{S}_{\eta} u\right),\left(u^{\prime}, 0\right)\right) & =m_{p}(\varepsilon)\left((u, 0),\left(u^{\prime}, 0\right)\right)-m_{p}(\varepsilon)\left(\left(u^{\prime}, 0\right),\left(0, \mathcal{S}_{\eta} u\right)\right) \\
& =m_{p}(\varepsilon)\left((u, 0),\left(u^{\prime}, 0\right)\right)-m_{p}(\varepsilon)\left(\left(0, \mathcal{S}_{\eta} u^{\prime}\right),\left(0, \mathcal{S}_{\eta} u\right)\right) .
\end{aligned}
$$


Furthermore, assumption $\left(\mathbf{H}_{3}\right)$ and definition (73) imply

$$
\begin{aligned}
\kappa|u|_{H_{\Gamma_{m D}}^{1}(\Omega)^{3}}^{2} & \leq m_{p}(\varepsilon)\left(\left(u, \mathcal{S}_{\eta} u\right),\left(u, \mathcal{S}_{\eta} u\right)\right)=m_{p}(\varepsilon)\left(\left(u, \mathcal{S}_{\eta} u\right),(u, 0)\right) \\
& \leq|M|_{L^{\infty}(\Omega, \mathcal{L}(\mathcal{H}))}\left(|u|_{H_{\Gamma_{m}, D}^{1}(\Omega)^{3}}+\left|\mathcal{S}_{\eta} u\right|_{H_{\Gamma_{e}, D}^{1}}(\Omega)|u|_{H_{\Gamma_{m, D}}^{1}}(\Omega)^{3}\right. \\
& \leq|M|_{L^{\infty}(\Omega, \mathcal{L}(\mathcal{H}))}\left(1+\frac{|M|_{L}^{\infty}(\Omega, \mathcal{H})}{\kappa}\right)|u|_{H_{\Gamma_{m}, D}^{1}}^{2}(\Omega)^{3}
\end{aligned}
$$

which proves that $\ll \cdot, \gg_{\eta}$ generates an Hilbert structure on $\mathbf{U}_{\eta}$ equivalent to the usual one. Now, the definitions of $\mathrm{A}_{\eta}$ and $\ll \cdot, \gg_{\eta}$ imply

$$
\ll \mathrm{U}, \mathrm{A}_{\eta} \mathrm{U} \gg_{\eta}=\ll(u, v),(v, z) \gg_{\eta}=m_{p}(\varepsilon)\left(\left(u, \mathcal{S}_{\eta} u\right),(v, 0)\right)+<v, z>_{\eta}=0 .
$$

Then, to establish that the skew-symmetric operator $\mathrm{A}_{\eta}$ is skew-adjoint, it suffices to show that for all $\mathbf{F}=\left(f_{1}, f_{2}\right)$ in $\mathbf{U}_{\eta}$ and all $\lambda>0$, the equation $\mathrm{U}-\lambda \mathrm{A}_{\eta}=\mathrm{F}$ has a unique solution $\mathrm{U}=(u, v)$. The definition of $\mathrm{A}_{\eta} \mathrm{U}=(v, z)$ implies $u-\lambda^{2} z=f_{1}+\lambda f_{2}$ and (74) yields

$$
<u, w>_{\eta}+\lambda^{2} m_{p}(\varepsilon)\left(\left(u, \mathcal{S}_{\eta} u\right),(w, 0)\right)=<f_{1}+\lambda f_{2}, w>_{\eta}, \forall w \in H_{\Gamma_{m D}}^{1}(\Omega)^{3} .
$$

The Lax-Milgram lemma implies the existence and uniqueness of a solution $\bar{u}$ of $(80)$ and, clearly, $\overline{\mathrm{U}}=\left(\bar{u},\left(\bar{u}-f_{1}\right) / \lambda\right)$ belongs to $D\left(\mathrm{~A}_{\eta}\right)$ and solves $\overline{\mathrm{U}}-\lambda \mathrm{A}_{\eta} \overline{\mathrm{U}}=F$.

The proof is achieved by invoking the Stone theorem and the fact that $(72)$ implies $\mathrm{F}(\eta) \in C^{0}\left([0, T] ; \mathrm{U}_{\eta}\right)$.

Eventually, this proposition and the regularity property (72) imply the

Theorem 3. Under assumptions $\left(\boldsymbol{H}_{3}\right),\left(\boldsymbol{H}_{4}\right),\left(\boldsymbol{H}_{7}\right)$ and $\left(\boldsymbol{H}_{8}\right)$, the problem $\mathcal{Q}(\eta, \Omega)_{p}$ has a unique solution of class $C^{1}\left([0, T] ; \boldsymbol{Y}_{\eta}\right)$.

A similar result is shown in [8].

Remark 3. By modifying the definition of $s^{e}$, it is possible to choose body forces $f_{\eta}$ in $W^{1, \infty}\left([0, T] ; L^{2}(\Omega)^{3}\right)$. This milder assumption leads to technical complications in the asymptotic analysis which are exposed in [18]. In order to clarify the matter, a more direct method is chosen here.

\section{The various asymptotic behaviors}

We will treat independently the behavior of $y^{e}(\eta)$ and the one of $y^{r}(\eta)$. As a solution of a quasi-static problem, $y^{e}(\eta)$ has an asymptotic behavior described by the results obtained in the preceding section. On the other hand, we choose the framework of the theory of Trotter (see [16]) of approximation of semigroups of operators acting on variable spaces to deal with the asymptotic behavior of $y^{r}(\eta)$. We will consider four cases, indexed by $q$, of relative behaviors of the parameters $\varepsilon$ and $\rho$ :

$$
q=1: \rho \rightarrow \bar{\rho} \in] 0,+\infty\left[, q=2: \rho \rightarrow 0 \text { and } \rho / \varepsilon^{2} \rightarrow+\infty, q=3: \rho / \varepsilon^{2} \rightarrow \overline{\bar{\rho}} \in\right] 0,+\infty\left[, q=4: \rho=o\left(\varepsilon^{2}\right)\right.
$$

Lastly, we make the assumption of convergence and regularity

$\left(\mathbf{H}_{9}\right):\left\{\begin{array}{c}\cdot\left(f_{\eta}, g_{\eta}, F_{\eta}, d_{\eta}, \varphi_{0_{\eta}}, k\left(\varepsilon, \varphi_{0_{\eta}}\right)\right) \rightarrow\left(f_{\bar{\eta}}, g_{\bar{\eta}}, F_{\bar{\eta}}, d_{\bar{\eta}}, \varphi_{0_{\bar{\eta}}}, k_{0}\right) \text { strongly in } \\ W^{2,1}\left((0, T), L^{2}(\Omega)^{3} \times L^{2}\left(\Gamma_{m N}\right)^{3} \times L^{2}(\Omega) \times L^{2}\left(\Gamma_{e N}\right) \times L^{2}(\Omega) \times L^{2}(\Omega, \mathcal{H})\right), \text { with }\left(k_{0}\right)_{p}^{0}=\left(k\left(0, \varphi_{0_{\eta}}\right)\right)_{p}^{0}, \\ \cdot\left(f_{\bar{\eta}}, g_{\bar{\eta}}, F_{\bar{\eta}}, d_{\bar{\eta}}, \varphi_{0_{\bar{\eta}}}\right) \in C^{2,1}\left([0, T], L^{2}\left(\Gamma_{m N}\right)^{3} \times L^{2}(\Omega) \times L^{2}\left(\Gamma_{e N}\right) \times H^{1}(\Omega)\right) \text { if } q=1,3 . \\ \cdot \frac{\sqrt{\rho}}{\varepsilon}\left(u^{e}(\eta)\right)_{3} \text { converges to } 0 \text { in } W^{2,1}\left([0, T], L^{2}(\Omega)\right) \text { if } q=1,2 .\end{array}\right.$

Remark 4. The last point in $\left(\mathbf{H}_{9}\right)$ concerns the magnitude and the nature of the external loading: it requires that the scaled kinetic energy of the quasi-static response does not tend to infinity. 
Due to the pointwise limits of the scaled kinetic energy (see (69)), we will see that there are no flexural motions when $q=1,2$. Thus, we introduce the following spaces of limit electromechanical states:

$$
\mathbf{S}_{p, q}:=\mathbf{V}_{K L}^{M} \times \Phi_{p}, q=1,2, \quad \mathbf{S}_{p, q}:=\mathbf{V}_{K L} \times \Phi_{p}, q=3,4
$$

and the limit continuous linear form on $\mathbf{S}_{p, q}$ :

$$
L_{\bar{\eta}}(r ; t):=\int_{\Omega} f_{\bar{\eta}}(t) \cdot w d x+\int_{\Gamma_{m N}} g_{\bar{\eta}}(t) \cdot w d s+\int_{\Omega} F_{\bar{\eta}}(t) \psi d x+\int_{\Gamma_{e N}} d_{\bar{\eta}}(t) \psi d s
$$

Asymptotic behavior of $s^{e}(\eta)$ Combining Corollary 1 with an equicontinuity argument, we deduce the:

Proposition 3. Under assumptions $\left(\boldsymbol{H}_{3}\right)-\left(\boldsymbol{H}_{5}\right),\left(\boldsymbol{H}_{7}\right)$ and $\left(\boldsymbol{H}_{9}\right)$, when $\eta$ goes to $\bar{\eta}$, the family $\left(s^{e}(\eta)(t)\right)_{\eta}$ of the unique solutions of $\mathcal{P}^{e}(\eta, \Omega)_{p}$ strongly converges in $\boldsymbol{X}_{p}$ to $\bar{s}^{e} \in C^{2,1}\left([0, T] ; \boldsymbol{X}_{p}\right)$, the unique solution of:

$$
\text { Find } s \in\left(0, \varphi_{0}\right)+\boldsymbol{S}_{p, q} \text { such that } \tilde{m}_{p}(s, r)=L_{\bar{\eta}}(r ; t), \forall(r, t) \in \boldsymbol{S}_{p, q} \times[0, T] \text {, }
$$

uniformly on $[0, T]$. In addition, $\lim _{\eta \rightarrow \bar{\eta}} m_{p}(\varepsilon)\left(s_{\eta}^{e}(t), s_{\eta}^{e}(t)\right)=\widetilde{m}_{p}\left(s^{e}(t), s^{e}(t)\right)$ uniformly on $[0, T]$.

Remark 5. Assumption $\left(\mathbf{H}_{9}\right)$ implies that $\bar{u}_{3}^{e}=0$ when $q=1,2$.

Asymptotic behavior of $s^{r}(\boldsymbol{\eta})$ Because the electrical potential is not concerned by the evolution equations, we first study the asymptotic behavior of $\mathrm{U}^{r}(\eta)$.

Operational framework Again, because of the various limit behaviors of the scaled kinetic energy, we introduce the spaces:

$$
\begin{aligned}
& \mathbf{V}_{1}:=\mathbf{V}_{K L}^{M}, \mathbf{V}_{3}:=\mathbf{V}_{K L}^{F} \\
& \mathbf{K}_{1}:=\left\{v \in L^{2}(\Omega)^{3}: v=\left(v_{1}, v_{2}, 0\right), v_{\alpha}(x)=v_{\alpha}(\widehat{x}), v_{\alpha} \in L^{2}(\omega)\right\}, \mathbf{K}_{3}:=\left\{v \in H^{-1}(\Omega)^{3}: \exists \eta \in L^{2}(\omega), v_{\alpha}=-x_{3} \partial_{\alpha} \eta, v_{3}=\eta\right\}
\end{aligned}
$$

the last two spaces being respectively equipped with the scalar products:

$$
K_{1}(v, w):=\bar{\rho} \int_{\Omega} \widehat{v} \cdot \widehat{w} \delta(x) d x, \quad K_{3}(v, w):=\overline{\bar{\rho}} \int_{\Omega} v_{3} w_{3} \delta(x) d x
$$

The limit spaces of the "dynamical part" of the mechanical phases will be:

$$
\mathbf{U}_{q}:=\mathbf{V}_{q} \times \mathbf{K}_{q}
$$

Remark 6 . In the cases $q=2$ and $q=4$ we will see that the asymptotic behavior is purely quasi-static. This explains why the spaces $\mathbf{U}_{q}$ are defined only when $q=1$ and $q=3$.

As in (73), we define a linear, continuous and one to one operator:

$$
\mathcal{S}: u \in \mathbf{V}_{K L} \mapsto \mathcal{S} u:=\varphi \in \Phi_{p} \quad ; \quad \widetilde{m}_{p}((u, \varphi),(0, \psi))=0, \forall \psi \in \Phi_{p},
$$

which permits us to equip $\mathbf{U}_{q}$ with an hilbertian structure through the scalar product

$$
\ll \mathrm{U}, \mathrm{U}^{\prime} \gg_{p, q}:=\widetilde{m}_{p}\left((u, \mathcal{S} u),\left(u^{\prime}, 0\right)\right)+k_{q}\left(v, v^{\prime}\right) .
$$

We denote the associated norm by $\|\cdot\|_{p, q}$. 
Remark \%. It should be noted that $\mathcal{S}$ maps $\mathbf{V}_{K L}^{M}$ in $\Phi_{1}, \Phi_{2}^{o}$ and $\mathbf{V}_{K L}^{F}$ in $\{0\}, \Phi_{2}^{e}$, if $p=1,2$ respectively. This rises from the decoupling assumption $\left(\mathbf{H}_{p}^{\text {decoupl }}\right)$ and (55)

The study of the convergence of the phases requires the introduction of an operator $\mathrm{Q}_{\eta}$ which makes it possible to compare the elements of $\mathbf{U}_{\eta}$ with those of $\mathbf{U}_{q}$ :

$$
\left\{\begin{array}{l}
\mathrm{Q}_{\eta}: \mathrm{U} \in \mathbf{U}_{q}=(u, v) \mapsto \mathrm{Q}_{\eta} \mathrm{U}:=\left(u_{\eta}, v_{\eta}\right) \in \mathbf{U}_{\eta} \text { such that: } \\
\cdot m_{p}(\varepsilon)\left(\left(u_{\eta}, \mathcal{S}_{\eta} u_{\eta}\right),(w, 0)\right)=\widetilde{m}_{p}((u, \mathcal{S} u),(w, 0)), \forall w \in H_{\Gamma_{m D}}^{1}(\Omega)^{3} \\
\cdot v_{\eta}=\bar{\rho} v / \rho \text { if } q=1 \text { and } v_{\eta}=\overline{\bar{\rho}} \varepsilon^{2} v_{3} / \rho \text { if } q=3 .
\end{array}\right.
$$

Note that $\ll \mathrm{Q}_{\eta} \mathrm{U}, \mathrm{U}^{\prime} \gg_{\eta}=\ll \mathrm{U}, \mathrm{U}^{\prime} \gg_{p, q}$ for all $\mathrm{U}$ in $\mathbf{U}_{q}$ and all $\mathrm{U}^{\prime}$ in $\mathbf{U}_{\eta}$. As a simple consequence of Corollary 2 with $\Lambda(r)=\widetilde{m}_{p}((u, \mathcal{S} u),(w, 0))$, for all $r=(w, \psi)$ in $\mathbf{S}_{\eta}$, we have the fundamental

Proposition 4. The spaces $\boldsymbol{U}_{\eta}$ associated with the operators $\mathrm{Q}_{\eta}$ form a sequence of Hilbert spaces approximating $\boldsymbol{U}_{q}$ in the sense of Trotter:

i) $\exists C \in \mathbb{R}^{+}$such that $\left\|\mathrm{Q}_{\eta} \mathrm{U}\right\|_{\eta} \leq C\|\mathrm{U}\|_{p, q}, \forall \mathrm{U} \in \boldsymbol{U}_{q}$.

ii) $\lim _{\eta \rightarrow \bar{\eta}}\left\|\mathrm{Q}_{\eta} \mathrm{U}\right\|_{\eta}=\|\mathrm{U}\|_{p, q}, \forall \mathrm{U} \in \boldsymbol{U}_{q}$.

Moreover, if $\mathrm{U}=(u, v), \mathrm{Q}_{\eta} \mathrm{U}=\left(\widetilde{u}_{\eta}, \widetilde{v}_{\eta}\right), \mathrm{U}_{\eta}=\left(u_{\eta}, v_{\eta}\right)$ then, when $\eta$ goes to $\bar{\eta}$ :

iii) $\widetilde{u}_{\eta} \rightarrow u$ in $H^{1}(\Omega)$ and $\widetilde{v}_{\eta} \rightarrow v$ in $L^{2}(\Omega)^{3}$ when $q=1,\left(\widetilde{v}_{\eta}\right)_{3} \rightarrow v_{3}$ in $L^{2}(\Omega)$ when $q=3$.

iv) $\lim _{\eta \rightarrow \bar{\eta}}\left\|\mathrm{Q}_{\eta} \mathrm{U}-\mathrm{U}_{\eta}\right\|_{\eta}=0 \Leftrightarrow\left\{\begin{array}{l}\left\|\mathrm{U}_{\eta}\right\|_{\eta} \rightarrow\|\mathrm{U}\|_{p, q}, u_{\eta} \rightarrow u \text { in } H^{1}(\Omega)^{3} \text { and } \\ v_{\eta} \rightarrow v \text { in } L^{2}(\Omega)^{3} \text { when } q=1 \\ \left(v_{\eta}\right)_{3} \rightarrow v_{3} \text { in } L^{2}(\Omega) \text { and } \\ \varepsilon v_{\eta} \rightarrow 0 \text { in } L^{2}(\Omega) \text { when } q=3 .\end{array}\right.$

The limit evolution equation In the cases $q=1$ and $q=3$, the limit problem will involve an evolution equation similar to (75) but governed by the following operator:

$$
\left\{\begin{array}{l}
D(\mathrm{~A})=\left\{\mathrm{U}=(u, v) \in \mathbf{U}_{p}: v \in \mathbf{V}_{q}, \exists ! z \in \mathbf{K}_{q} \quad \text { such that } K_{q}(z, w)+\widetilde{m}_{p}((u, \mathcal{S} u),(w, 0))=0, \forall w \in \mathbf{V}_{q}\right\} \\
\mathrm{A} \mathbf{U}=(v, z)
\end{array}\right.
$$

Arguing as in the proof of the Proposition 2 yields the

Proposition 5. The operator A is skew-adjoint.

Hence, similarly to the Proposition 2, with the compatibility assumption between initial limit loading and mechanical phase

$$
\left(\mathbf{H}_{10}\right): \mathrm{U}^{0}-\mathrm{U}^{e}(0) \in D(\mathrm{~A})
$$

we have the

Proposition 6. Under hypotheses $\left(\boldsymbol{H}_{1}\right)-\left(\boldsymbol{H}_{5}\right),\left(\boldsymbol{H}_{7}\right)-\left(\boldsymbol{H}_{10}\right)$ and $\left(\boldsymbol{H}_{p}^{\text {decoupl }}\right)$, the evolution equation

$$
\dot{\mathrm{U}}^{r}-\mathrm{AU}^{r}=\mathrm{F}^{r}:=\left(0,-\ddot{u}^{e}\right), \quad \mathrm{U}^{r}(0)=\mathrm{U}^{0}-\mathrm{U}^{e}(0),
$$

has a unique solution $\bar{U}^{r}:=\left(\bar{u}^{r}, \bar{v}^{r}\right)$ of class $C^{1}\left([0, T] ; \boldsymbol{U}_{q}\right)$. 
Convergence of $\mathbf{U}^{\boldsymbol{r}}(\boldsymbol{\eta})$ The additional assumption:

$$
\left(\mathbf{H}_{11}\right): \lim _{\eta \rightarrow \bar{\eta}}\left\|\mathrm{Q}_{\eta} \mathrm{U}^{0}-\mathrm{U}^{0}(\eta)\right\|_{\eta}=0, q=1,3 ; \lim _{\eta \rightarrow \bar{\eta}}\left\|\mathrm{U}_{\eta}^{0}-\mathrm{U}_{\eta}^{e}(0)\right\|_{\eta}=0, q=2,4
$$

allows us to state the

Proposition 7. Under hypotheses $\left(\boldsymbol{H}_{1}\right)-\left(\boldsymbol{H}_{11}\right)$ we have

$$
\lim _{\eta \rightarrow \bar{\eta}}\left\|\mathrm{Q}_{\eta} \mathrm{U}^{r}-\mathrm{U}^{r}(\eta)\right\|_{\eta}=0, q=1,3 ; \lim _{\eta \rightarrow \bar{\eta}}\left\|\mathrm{U}^{r}(\eta)\right\|_{\eta}=0, q=2,4
$$

Proof. Cases $q=1$ and $q=3$ :

Within the framework of the Trotter theory of approximation of semigroups of operators acting on variable spaces ( $c f$. [16]), three conditions has to be satisfied to obtain the convergence results. These conditions of convergence in the sense of Trotter of the resolvents, the initial conditions and the second members read as:

$$
\begin{aligned}
& \lim _{\eta \rightarrow \bar{\eta}}\left\|\left(I-\lambda \mathrm{A}_{\eta}\right)^{-1} \mathrm{Q}_{\eta} \mathrm{V}-\mathrm{Q}_{\eta}(I-\lambda \mathrm{A})^{-1} \mathrm{~V}\right\|_{\eta}=0, \forall(\lambda, \mathrm{V}) \in \mathbb{R}^{+} \times \mathbf{U}_{q}, \\
& \lim _{\eta \rightarrow \bar{\eta}}\left\|\mathrm{Q}_{\eta} \mathrm{U}^{r}(0)-\mathrm{U}^{r}(\eta)(0)\right\|_{\eta}=0, \\
& \lim _{\eta \rightarrow \bar{\eta}} \int_{0}^{T}\left\|\mathrm{Q}_{\eta} \mathrm{F}^{r}-\mathrm{F}^{r}(\eta)\right\|_{\eta} d t=0
\end{aligned}
$$

Let $\mathrm{V}:=(\mathcal{V}, \mathcal{W}), \mathbf{Q}_{\eta} \mathrm{V}:=(\mathcal{V}(\eta), \mathcal{W}(\eta)), \mathrm{U}(\eta)=\left(u_{\eta}, v_{\eta}\right)=\left(I-\lambda \mathrm{A}_{\eta}\right)^{-1} \mathrm{Q}_{\eta} \mathrm{V}$ and $\mathrm{U}=(I-\lambda \mathrm{A})^{-1} \mathrm{~V}$, then:

$$
\left\{\begin{array}{l}
<v_{\eta}, w>_{\eta}+\lambda^{2} m_{p}(\varepsilon)\left(\left(v_{\eta}, \mathcal{S}_{\eta} v_{\eta}\right),(w, 0)=\ll \mathrm{Q}_{\eta} \mathrm{V},(-\lambda w, w) \gg_{\eta}=\ll \mathrm{V},(-\lambda w, w) \gg_{p, q}, \forall w \in H_{\Gamma_{m D}}^{1}(\Omega)^{3}\right. \\
u_{\eta}=\mathcal{V}_{\eta}+\lambda v_{\eta}
\end{array}\right.
$$

and

$$
\left\{\begin{array}{l}
k_{q}(v, w)+\lambda^{2} \widetilde{m}_{p}((v, \mathcal{S} v),(w, 0))=\ll \mathrm{V},(-\lambda w, w) \gg_{p, q}, \forall w \in \mathbf{V}_{q} \\
u=\mathcal{V}+\lambda v
\end{array}\right.
$$

Hence $(94)_{1}$ is a consequence of Corollary 2 with $\ll \mathrm{V},(-\lambda w, w) \gg_{p, q}$ as second member and of the point $(i v)$ in Proposition 4 . Lastly, $(94)_{2}$ is given by $\left(\mathbf{H}_{11}\right)$, while $(94)_{3}$ stems from $(92),(90)$ and $\left(\mathbf{H}_{9}\right)$.

\section{Cases $q=2$ and $q=4$ :}

Through the Duhamel formula, hypotheses $\left(\mathbf{H}_{9}\right)$ and $\left(\mathbf{H}_{11}\right)$ clearly imply that $\left\|\mathbf{U}^{r}(\eta)(t)\right\|_{\eta}$ uniformly converges to 0 on $[0, T]$ when $\eta$ goes to $\bar{\eta}$.

Consequence: convergence of $\boldsymbol{s}^{\boldsymbol{r}}(\boldsymbol{\eta})$ Let $s^{r}(\eta):=\left(u^{r}(\eta), \mathcal{S}_{\eta} u^{r}(\eta)\right)$ and $\bar{s}^{r}:=\left(\bar{u}^{r}, \mathcal{S} u^{r}\right), \bar{u}^{r}=0$ if $q=2,4$. Then, the Proposition 7 and Corollary 1 with $\Lambda_{\varepsilon}(w, \psi)=\widetilde{m}_{p}((u, \mathcal{S} u),(w, 0))$ yields the

Proposition 8. When $\eta$ goes to $\bar{\eta}$ we have:

$$
\left|s^{r}(\eta)(t)-\bar{s}^{r}(t)\right|_{\boldsymbol{x}_{p}} \rightarrow 0, \quad m_{p}(\varepsilon)\left(s^{r}(\eta)(t), s^{r}(\eta)(t)\right) \rightarrow \widetilde{m}_{p}\left(\bar{s}^{r}(t), \bar{s}^{r}(t)\right),
$$

uniformly on $[0, T]$. 


\subsection{Final results}

We can now synthetize all the convergence results obtained previously in the

Theorem 4. Under assumptions $\left(\boldsymbol{H}_{3}\right)-\left(\boldsymbol{H}_{11}\right)$ and $\left(\boldsymbol{H}_{p}^{\text {decoupl }}\right)$, when $\eta$ goes to $\bar{\eta}$, the family $\left(s_{\eta}\right)_{\eta}$ strongly converges in $\boldsymbol{X}_{p}$ and uniformly on $[0, T]$ to the unique element $\bar{s}_{p, q}=\left(\bar{u}_{p, q}, \bar{\varphi}_{p, q}\right)$ of $C^{1}\left([0, T] ;\left(0, \varphi_{0}\right)+\boldsymbol{S}_{p, q}\right)$ satisfying:

$$
\left\{\begin{array}{l}
\text { if } q=2 \text { or } 4: \quad \widetilde{m}_{p}\left(\bar{s}_{p, q}, r\right)=L_{\bar{\eta}}(r), \forall(r, t) \in \boldsymbol{S}_{p, q} \times[0, T] \\
\text { if } q=1 \text { or } 3: K_{q}\left(\ddot{\bar{u}}_{p, q}, w\right)+\widetilde{m}_{p}\left(\bar{s}_{p, q}, r\right)=L_{\bar{\eta}}(r), \forall(r=(w, \psi), t) \in \boldsymbol{S}_{p, q} \times[0, T] \\
\left(\ddot{\bar{u}}_{p, q}(0), \dot{\bar{u}}_{p, q}(0)\right)=\mathrm{U}^{0},
\end{array}\right.
$$

$\left(\widehat{u}_{p, 1}\right)_{\alpha},\left(\bar{u}_{p, 3}\right)_{3}$ belongs to $C^{2}\left([0, T] ; L^{2}(\omega)\right), \bar{s}_{p, q}$ belongs to $C^{2}\left([0, T] ; \boldsymbol{X}_{p}\right)$ if $q=2$ or 4 . Moreover, $\lim _{\eta \rightarrow \bar{\eta}} m_{p}(\varepsilon)(s(\eta), s(\eta))=$ $\widetilde{m}_{p}\left(\bar{s}_{p, q}, \bar{s}_{p, q}\right)$ uniformly on $[0, T]$.

Proof. It remains to prove that $\lim _{\eta \rightarrow \bar{\eta}} m_{p}(\varepsilon)\left(s^{r}(\eta), s^{e}(\eta)\right)=\widetilde{m}_{p}\left(s^{r}, s^{e}\right)$. Arguing as in the proof of Proposition $1, k\left(\varepsilon, s^{e}(\eta)\right)$ converges strongly in $L^{2}(\Omega, \mathcal{H})$ to $\bar{k}^{e}$ with $\left(\bar{k}^{e}\right)_{p}^{0}=k\left(s^{e}\right)_{p}^{0}$. Proposition 7 implies that $k\left(\varepsilon, s^{r}(\eta)\right)$ converges strongly in $L^{2}(\Omega, \mathcal{H})$ to $\bar{k}^{r}$ which is also the limit of $k\left(\varepsilon,\left(\widetilde{u}^{r}(\eta), \mathcal{S} \widetilde{u}^{r}(\eta)\right)\right.$ where $\widetilde{u}^{r}(\eta)$ is the "displacement component" of $\mathrm{Q}_{\eta} \mathrm{U}^{r}$. Arguing again as in the proof of Proposition 1 when $L(w, \psi)$ is replaced by $\widetilde{m}_{p}\left(\left(u^{r}, \mathcal{S} \widetilde{u}^{r}(\eta)\right),(w, 0)\right)$ we deduce that $\left(M \bar{k}^{r}\right)_{p}^{0}=0$. The result stems from $\left(M \bar{k}^{r}\right)_{p}^{0}=\widetilde{M}\left(\bar{k}^{r}\right)_{p}^{0}$.

\section{A few comments}

In the cases $q=2$ and 4 , the relation $\lim _{\eta \rightarrow \bar{\eta}}\left\|\mathrm{U}^{r}(\eta)\right\|_{\eta}=0$ means that the limit response of the plate to the electromechanical loading is purely quasi-static. On the other hand, in the cases $q=1$ and $q=3$, the acceleration of the displacement is involved. Moreover, assumption $\left(\mathbf{H}_{p}^{\text {decoupl }}\right.$ ) implying that $\mathbf{S}_{p, q}$ is the direct sum of subspaces $\widetilde{m}_{p}$-polar, a decoupling between the membrane and the flexural displacements appears. Thus, if $q=1,2$ there is no flexion and the membrane response is dynamic if $q=1$, quasi-static if $q=2$. If $q=3,4$ the membrane response is quasi-static and the flexural response is dynamic if $q=3$ and quasi-static if $q=4$. In these last two cases, the limit electrical potential is not involved in the equation providing the flexural part if $p=1$.

\subsection{The suggested model}

For every value of $q$ we can, like in Section 2.6, come back to the real plate occupying $\overline{\Omega^{\varepsilon}}$ and set a problem solved by $\left(\Pi_{p}^{\varepsilon}\right)^{-1}\left(\bar{s}_{p, q}\right)$. But, since $\bar{\rho}$ and $\overline{\bar{\rho}}$ do not have any physical meaning, we prefer to propose the following model for the dynamic behavior of a plate of thickness $2 \varepsilon$ and with density $\rho \delta\left(\widehat{x}, x_{3} / \varepsilon\right)$ :

$$
\left\{\begin{array}{l}
\text { Find } \bar{s}_{p}^{\varepsilon}=\left(\bar{u}_{p}^{\varepsilon}, \bar{\varphi}_{0}^{\varepsilon}\right) \in \mathbf{S}_{p}^{\varepsilon} \text { such that } \int_{\Omega^{\varepsilon}} \rho \delta \ddot{\bar{u}}_{p}^{\varepsilon} \cdot w d x+\widetilde{m}_{p}^{\varepsilon}\left(\bar{s}_{p}^{\varepsilon}, r\right)=L^{\varepsilon}(r), \forall(r=(w, \psi), t) \in \mathbf{S}_{p}^{\varepsilon} \times[0, T] \\
\left(\bar{u}_{p}^{\varepsilon}(0), \dot{\bar{u}}_{p}^{\varepsilon}(0)\right)=\mathrm{U}_{0}^{\varepsilon}
\end{array}\right.
$$

where $\mathbf{U}_{0}^{\varepsilon}$ is the descaled of $\mathbf{U}_{0}$. Actually, with hypothesis $\left(\mathbf{H}_{p}^{\text {decoupl }}\right.$ ), it clearly appears (see (48)) that the main tendancy is given by the magnitudes of $\rho$ and $\varepsilon^{-2} \rho$.

\section{References}

1. M. Bernadou, C. Haenel, Contributions à l'analyse numérique des coques piézoélectriques, Proc. Coll. Calcul Structures, (1995) 841-846.

2. P. Bisegna, F. Maceri, A consistent theory of thin piezoelectric plates. J. Intelligent Mat. Syst. Struct. 7 (1996) $372-389$.

3. H. Brézis, Analyse fonctionnelle. Théorie et applications. (Masson, 1983).

4. P. G. Ciarlet, Mathematical Elasticity, Vol. II. (North Holland, 1997).

5. L. Costa, I. Figueiredo, R. Leal, P. Oliveira, G. Stadler, Modeling and numerical study of actuator and sensor effects for a laminated piezoelectric plate, Computers and Structures 85 (2007) 385-403.

6. M. Lenczner, Modèle d'assemblage de plaques piézoélectriques, Rapport d'activité du Laboratoire de Calcul Scientifique (1993), Université de Franche-Comté, Besançon, France. 
7. G. A. Maugin, D. Attou, An asymptotic theory of thin piezoelectric plates, Q. Jl. Mech. Appl. Math. 43 (1993) 519-524.

8. B. Miara, Contrôlabilité d'un corps piézoélectrique, C. R. Acad. Sci. Série I Paris 333 (2001) 267-270.

9. I. M. Narra Figueiredo, C. M. Franco Leal, A piezoelectric anisotropic plate model, Asymptotic Analysis 44 (2005), 327-346.

10. M. Rahmoune, A. Benjeddou, R. Ohayon, New thin piezoelectric plates models, J. Intelligent Mat. Syst. Struct. 9 (1998) 1017-1029.

11. A. Raoult, A. Sène, Modelling of piezoelectric plates including magnetic effects, Asymptotic Analysis 34 (2003) 1-40.

12. N. N. Rogacheva, The theory of piezoelectric shells and plates. (CRC Press, 1994).

13. D. Royer, E. Dieulesaint, Ondes élastiques dans les solides, Tome I. (Masson, 1996).

14. E. Sanchez-Palencia, Asymptotic and singular properties of a class of singular-stiff problems, J. Math. Pures Appl. 332 (2004) 519-524.

15. A. Sène, Modélisation asymptotique de plaques : Contrôlabilité exacte frontière, piézoélectricité. Ph. D. Thesis (1999) Université Joseph Fourier-Grenoble I, France.

16. H. F. Trotter, Approximation of semi-groups of operators, Pacific J. of Math. 28 (1958) 887-919.

17. T. Weller, C. Licht, Analyse asymptotique de plaques minces linéairement piézoélectriques, C. R. Acad. Sci. Série I Paris, 333 (2002) 267-270.

18. T. Weller, C. Licht, Réponse dynamique asymptotique de plaques minces linéairement piézoélectriques dans l'approximation quasi-électrostatique, C. R. Mécanique, 332 (2004) 519-524.

19. T. Weller, Études des symétries et modèles de plaques en piézoélectricité linéarisée. Ph. D. Thesis (2004) Université Montpellier II, France. 